\title{
From Theory to Practice: An Overview of MIMO Space-Time Coded Wireless Systems
}

\author{
David Gesbert, Member, IEEE, Mansoor Shafi, Fellow, IEEE, Da-shan Shiu, Member, IEEE, \\ Peter J. Smith, Member, IEEE, and Ayman Naguib, Senior Member, IEEE
}

Tutorial Paper

\begin{abstract}
This paper presents an overview of recent progress in the area of multiple-input-multiple-output (MIMO) space-time coded wireless systems. After some background on the research leading to the discovery of the enormous potential of MIMO wireless links, we highlight the different classes of techniques and algorithms proposed which attempt to realize the various benefits of MIMO including spatial multiplexing and space-time coding schemes. These algorithms are often derived and analyzed under ideal independent fading conditions. We present the state of the art in channel modeling and measurements, leading to a better understanding of actual MIMO gains. Finally, the paper addresses current questions regarding the integration of MIMO links in practical wireless systems and standards.
\end{abstract}

Index Terms-Beamforming, channel models, diversity, multiple-input-multiple-output (MIMO), Shannon capacity, smart antennas, space-time coding, spatial multiplexing, spectrum efficiency, third-generation (3G), wireless systems.

\section{INTRODUCTION}

D IGITAL communication using multiple-input-multipleoutput (MIMO), sometimes called a "volume-to-volume" wireless link, has recently emerged as one of the most significant technical breakthroughs in modern communications. The technology figures prominently on the list of recent technical advances with a chance of resolving the bottleneck of traffic capacity in future Internet-intensive wireless networks. Perhaps even more surprising is that just a few years after its invention the technology seems poised to penetrate large-scale standards-driven commercial wireless products and networks such as broadband wireless access systems, wireless local

Manuscript received June 1, 2002; revised December 5, 2002. The work of D. Gesbert was supported in part by Telenor AS, Norway.

D. Gesbert is with the Department of Informatics, University of Oslo, Blindern, 0316 Oslo, Norway (e-mail: gesbert@ifi.uio.no).

M. Shafi is with Telecom New Zealand, Wellington, New Zealand (e-mail: Mansoor.Shafi@telecom.co.nz).

D. Shiu is with Qualcomm, Inc., Campbell, CA 95008 USA (e-mail: dashiu@qualcomm.com).

P. J. Smith is with the Department of Electrical and Computer Engineering, University of Canterbury, Christchurch, New Zealand (e-mail: p.smith@elec.canterbury.ac.nz).

A. Naguib was with Morphics Technology, Inc., Campbell, CA 95008 USA He is now with Qualcomm, Inc., Campbell, CA 95008 USA.

Digital Object Identifier 10.1109/JSAC.2003.809458 area networks (WLAN), third-generation $(3 \mathrm{G})^{1}$ networks and beyond.

MIMO systems can be defined simply. Given an arbitrary wireless communication system, we consider a link for which the transmitting end as well as the receiving end is equipped with multiple antenna elements. Such a setup is illustrated in Fig. 1. The idea behind MIMO is that the signals on the transmit (TX) antennas at one end and the receive (RX) antennas at the other end are "combined" in such a way that the quality (bit-error rate or BER) or the data rate (bits/sec) of the communication for each MIMO user will be improved. Such an advantage can be used to increase both the network's quality of service and the operator's revenues significantly.

A core idea in MIMO systems is space-time signal processing in which time (the natural dimension of digital communication data) is complemented with the spatial dimension inherent in the use of multiple spatially distributed antennas. As such MIMO systems can be viewed as an extension of the so-called smart antennas, a popular technology using antenna arrays for improving wireless transmission dating back several decades.

A key feature of MIMO systems is the ability to turn multipath propagation, traditionally a pitfall of wireless transmission, into a benefit for the user. MIMO effectively takes advantage of random fading [1]-[3] and when available, multipath delay spread [4], [5], for multiplying transfer rates. The prospect of many orders of magnitude improvement in wireless communication performance at no cost of extra spectrum (only hardware and complexity are added) is largely responsible for the success of MIMO as a topic for new research. This has prompted progress in areas as diverse as channel modeling, information theory and coding, signal processing, antenna design and multiantenna-aware cellular design, fixed or mobile.

This paper discusses the recent advances, adopting successively several complementing views from theory to real-world network applications. Because of the rapidly intensifying efforts in MIMO research at the time of writing, as exemplified by the numerous papers submitted to this special issue of JSAC, a complete and accurate survey is not possible. Instead this paper forms a synthesis of the more fundamental ideas presented over the last few years in this area, although some very recent progress is also mentioned.

${ }^{1}$ Third-generation wireless UMTS-WCDMA. 


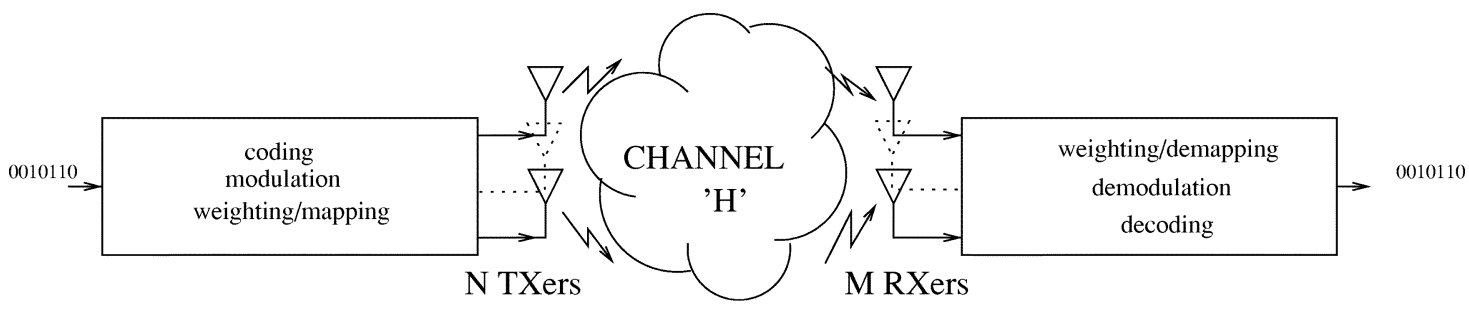

Fig. 1. Diagram of a MIMO wireless transmission system. The transmitter and receiver are equipped with multiple antenna elements. Coding, modulation, and mapping of the signals onto the antennas may be realized jointly or separately.

The article is organized as follows. In Section II, we attempt to develop some intuition in this domain of wireless research, we highlight the common points and key differences between MIMO and traditional smart antenna systems, assuming the reader is somewhat familiar with the latter. We comment on a simple example MIMO transmission technique revealing the unique nature of MIMO benefits. Next, we take an information theoretical stand point in Section III to justify the gains and explore fundamental limits of transmission with MIMO links in various scenarios. Practical design of MIMO-enabled systems involves the development of finite-complexity transmission/reception signal processing algorithms such as space-time coding and spatial multiplexing schemes. Furthermore, channel modeling is particularly critical in the case of MIMO to properly assess algorithm performance because of sensitivity with respect to correlation and rank properties. Algorithms and channel modeling are addressed in Sections IV and V, respectively. Standardization issues and radio network level considerations which affect the overall benefits of MIMO implementations are finally discussed in Section VI. Section VII concludes this paper.

\section{Principles of Space-Time (MIMO) Systems}

Consider the multiantenna system diagram in Fig. 1. A compressed digital source in the form of a binary data stream is fed to a simplified transmitting block encompassing the functions of error control coding and (possibly joined with) mapping to complex modulation symbols (quaternary phase-shift keying (QPSK), M-QAM, etc.). The latter produces several separate symbol streams which range from independent to partially redundant to fully redundant. Each is then mapped onto one of the multiple TX antennas. Mapping may include linear spatial weighting of the antenna elements or linear antenna space-time precoding. After upward frequency conversion, filtering and amplification, the signals are launched into the wireless channel. At the receiver, the signals are captured by possibly multiple antennas and demodulation and demapping operations are performed to recover the message. The level of intelligence, complexity, and a priori channel knowledge used in selecting the coding and antenna mapping algorithms can vary a great deal depending on the application. This determines the class and performance of the multiantenna solution that is implemented.

In the conventional smart antenna terminology, only the transmitter or the receiver is actually equipped with more than one element, being typically the base station (BTS), where the extra cost and space have so far been perceived as more easily affordable than on a small phone handset. Traditionally, the intelligence of the multiantenna system is located in the weight selection algorithm rather than in the coding side although the development of space-time codes (STCs) is transforming this view.

Simple linear antenna array combining can offer a more reliable communications link in the presence of adverse propagation conditions such as multipath fading and interference. A key concept in smart antennas is that of beamforming by which one increases the average signal-to-noise ratio (SNR) through focusing energy into desired directions, in either transmit or receiver. Indeed, if one estimates the response of each antenna element to a given desired signal, and possibly to interference signal(s), one can optimally combine the elements with weights selected as a function of each element response. One can then maximize the average desired signal level or minimize the level of other components whether noise or co-channel interference.

Another powerful effect of smart antennas lies in the concept of spatial diversity. In the presence of random fading caused by multipath propagation, the probability of losing the signal vanishes exponentially with the number of decorrelated antenna elements being used. A key concept here is that of diversity order which is defined by the number of decorrelated spatial branches available at the transmitter or receiver. When combined together, leverages of smart antennas are shown to improve the coverage range versus quality tradeoff offered to the wireless user [6].

As subscriber units (SU) are gradually evolving to become sophisticated wireless Internet access devices rather than just pocket telephones, the stringent size and complexity constraints are becoming somewhat more relaxed. This makes multiple antenna elements transceivers a possibility at both sides of the link, even though pushing much of the processing and cost to the network's side (i.e., BTS) still makes engineering sense. Clearly, in a MIMO link, the benefits of conventional smart antennas are retained since the optimization of the multiantenna signals is carried out in a larger space, thus providing additional degrees of freedom. In particular, MIMO systems can provide a joint transmit-receive diversity gain, as well as an array gain upon coherent combining of the antenna elements (assuming prior channel estimation).

In fact, the advantages of MIMO are far more fundamental. The underlying mathematical nature of MIMO, where data is transmitted over a matrix rather than a vector channel, creates new and enormous opportunities beyond just the added diversity or array gain benefits. This was shown in [2], where the 


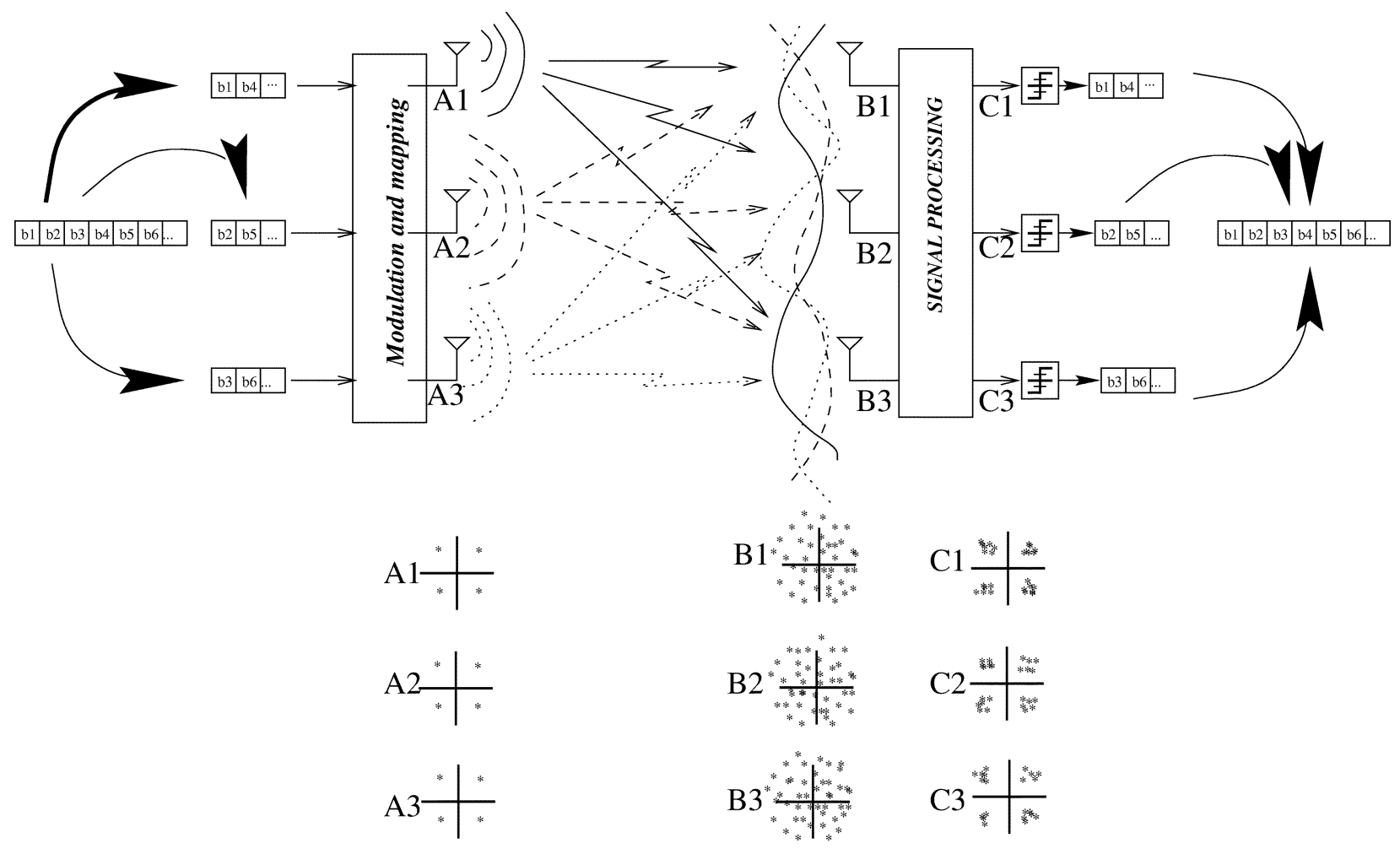

Fig. 2. Basic spatial multiplexing (SM) scheme with three TX and three RX antennas yielding three-fold improvement in spectral efficiency. Ai, Bi, and Ci represent symbol constellations for the three inputs at the various stages of transmission and reception.

author shows how one may under certain conditions transmit $\min (M, N)$ independent data streams simultaneously over the eigenmodes of a matrix channel created by $N$ TX and $M$ RX antennas. A little known yet earlier version of this ground breaking result was also released in [7] for application to broadcast digital TV. However, to our knowledge, the first results hinting at the capacity gains of MIMO were published by Winters in [8].

Information theory can be used to demonstrate these gains rigorously (see Section III). However, intuition is perhaps best given by a simple example of such a transmission algorithm over MIMO often referred to in the literature as V-BLAST ${ }^{2}$ [9], [10] or more generically called here spatial multiplexing.

In Fig. 2, a high-rate bit stream (left) is decomposed into three independent $1 / 3$-rate bit sequences which are then transmitted simultaneously using multiple antennas, thus consuming one third of the nominal spectrum. The signals are launched and naturally mix together in the wireless channel as they use the same frequency spectrum. At the receiver, after having identified the mixing channel matrix through training symbols, the individual bit streams are separated and estimated. This occurs in the same way as three unknowns are resolved from a linear system of three equations. This assumes that each pair of transmit receive antennas yields a single scalar channel coefficient, hence flat fading conditions. However, extensions to frequency selective cases are indeed possible using either a straightforward multiple-carrier approach (e.g., in orthogonal frequency division multiplexing (OFDM), the detection is performed over each flat subcarrier) or in the time domain by combining the MIMO space-time detector with an equalizer

\footnotetext{
${ }^{2}$ Vertical-Bell Labs Layered Space-Time Architecture.
}

(see for instance [11]-[13] among others). The separation is possible only if the equations are independent which can be interpreted by each antenna "seeing" a sufficiently different channel in which case the bit streams can be detected and merged together to yield the original high rate signal. Iterative versions of this detection algorithm can be used to enhance performance, as was proposed in [9] (see later in this paper for more details or in [14] of this special issue for a comprehensive study).

A strong analogy can be made with code-division multiple-access (CDMA) transmission in which multiple users sharing the same time/frequency channel are mixed upon transmission and recovered through their unique codes. Here, however, the advantage of MIMO is that the unique signatures of input streams ("virtual users") are provided by nature in a close-to-orthogonal manner (depending however on the fading correlation) without frequency spreading, hence at no cost of spectrum efficiency. Another advantage of MIMO is the ability to jointly code and decode the multiple streams since those are intended to the same user. However, the isomorphism between MIMO and CDMA can extend quite far into the domain of receiver algorithm design (see Section IV).

Note that, unlike in CDMA where user's signatures are quasi-orthogonal by design, the separability of the MIMO channel relies on the presence of rich multipath which is needed to make the channel spatially selective. Therefore, MIMO can be said to effectively exploit multipath. In contrast, some smart antenna systems (beamforming, interference rejection-based) will perform better in line-of-sight (LOS) or close to LOS conditions. This is especially true when the optimization criterion depends explicitly on angle of arrival/departure 
parameters. Alternatively, diversity-oriented smart antenna techniques perform well in nonline-of-sight (NLOS), but they really try to mitigate multipath rather than exploiting it.

In general, one will define the rank of the MIMO channel as the number of independent equations offered by the above mentioned linear system. It is also equal to the algebraic rank of the $M \times N$ channel matrix. Clearly, the rank is always both less than the number of TX antennas and less than the number of $\mathrm{RX}$ antennas. In turn, following the linear algebra analogy, one expects that the number of independent signals that one may safely transmit through the MIMO system is at most equal to the rank. In the example above, the rank is assumed full (equal to three) and the system shows a nominal spectrum efficiency gain of three, with no coding. In an engineering sense, however, both the number of transmitted streams and the level of BER on each stream determine the link's efficiency (goodput ${ }^{3}$ per TX antenna times number of antennas) rather than just the number of independent input streams. Since the use of coding on the multiantenna signals (a.k.a. space-time coding) has a critical effect on the BER behavior, it becomes an important component of MIMO design. How coding and multiplexing can be traded off for each other is a key issue and is discussed in more detail in Section IV.

\section{MIMO INFORMATION THEORY}

In Sections I and II, we stated that MIMO systems can offer substantial improvements over conventional smart antenna systems in either quality-of-service (QoS) or transfer rate in particular through the principles of spatial multiplexing and diversity. In this section, we explore the absolute gains offered by MIMO in terms of capacity bounds. We summarize these results in selected key system scenarios. We begin with fundamental results which compare single-input-single-output (SISO), single-input-multiple-output (SIMO), and MIMO capacities, then we move on to more general cases that take possible a priori channel knowledge into account. Finally, we investigate useful limiting results in terms of the number of antennas or SNR. We bring the reader's attention on the fact that we focus here on single user forms of capacity. A more general multiuser case is considered in [15]. Cellular MIMO capacity performance has been looked at elsewhere, taking into account the effects of interference from either an information theory point of view [16], [17] or a signal processing and system efficiency point of view [18], [19] to cite just a few example of contributions, and is not treated here.

\section{A. Fundamental Results}

For a memoryless $1 \times 1$ (SISO) system the capacity is given by

$$
C=\log _{2}\left(1+\rho|h|^{2}\right) \quad \mathrm{b} / \mathrm{s} / \mathrm{Hz}
$$

where $h$ is the normalized complex gain of a fixed wireless channel or that of a particular realization of a random channel. In (1) and subsequently, $\rho$ is the SNR at any RX antenna. As we deploy more RX antennas the statistics of capacity improve and

\footnotetext{
${ }^{3}$ The goodput can be defined as the error-free fraction of the conventional physical layer throughput.
}

with $M \mathrm{RX}$ antennas, we have a SIMO system with capacity given by

$$
C=\log _{2}\left(1+\rho \sum_{i=1}^{M}\left|h_{i}\right|^{2}\right) \quad \mathrm{b} / \mathrm{s} / \mathrm{Hz}
$$

where $h_{i}$ is the gain for RX antenna $i$. Note the crucial feature of (2) in that increasing the value of $M$ only results in a logarithmic increase in average capacity. Similarly, if we opt for transmit diversity, in the common case, where the transmitter does not have channel knowledge, we have a multipleinput-single-output (MISO) system with $N$ TX antennas and the capacity is given by [1]

$$
C=\log _{2}\left(1+\frac{\rho}{N} \sum_{i=1}^{N}\left|h_{i}\right|^{2}\right) \quad \mathrm{b} / \mathrm{s} / \mathrm{Hz}
$$

where the normalization by $N$ ensures a fixed total transmitter power and shows the absence of array gain in that case (compared to the case in (2), where the channel energy can be combined coherently). Again, note that capacity has a logarithmic relationship with $N$. Now, we consider the use of diversity at both transmitter and receiver giving rise to a MIMO system. For $N$ TX and $M$ RX antennas, we have the now famous capacity equation [1], [3], [21]

$$
C_{\mathrm{EP}}=\log _{2}\left[\operatorname{det}\left(\mathbf{I}_{M}+\frac{\rho}{N} \mathbf{H} \mathbf{H}^{*}\right)\right] \quad \mathrm{b} / \mathrm{s} / \mathrm{Hz}
$$

where $(*)$ means transpose-conjugate and $\mathbf{H}$ is the $M \times N$ channel matrix. Note that both (3) and (4) are based on $N$ equal power (EP) uncorrelated sources, hence, the subscript in (4). Foschini [1] and Telatar [3] both demonstrated that the capacity in (4) grows linearly with $m=\min (M, N)$ rather than logarithmically [as in (3)[. This result can be intuited as follows: the determinant operator yields a product of $\min (M, N)$ nonzero eigenvalues of its (channel-dependent) matrix argument, each eigenvalue characterizing the SNR over a so-called channel eigenmode. An eigenmode corresponds to the transmission using a pair of right and left singular vectors of the channel matrix as transmit antenna and receive antenna weights, respectivelly. Thanks to the properties of the log, the overall capacity is the sum of capacities of each of these modes, hence the effect of capacity multiplication. Note that the linear growth predicted by the theory coincides with the transmission example of Section II. Clearly, this growth is dependent on properties of the eigenvalues. If they decayed away rapidly then linear growth would not occur. However (for simple channels), the eigenvalues have a known limiting distribution [22] and tend to be spaced out along the range of this distribution. Hence, it is unlikely that most eigenvalues are very small and the linear growth is indeed achieved.

With the capacity defined by (4) as a random variable, the issue arises as to how best to characterize it. Two simple summaries are commonly used: the mean (or ergodic) capacity [3], [21], [23] and capacity outage [1], [24]-[26]. Capacity outage measures (usually based on simulation) are often denoted $C_{0.1}$ or $C_{0.01}$, i.e., those capacity values supported $90 \%$ or $99 \%$ of the time, and indicate the system reliability. A full description of the capacity would require the probability density function 
or equivalent. Some results are available here [27] but they are limited.

Some caution is necessary in interpreting the above equations. Capacity, as discussed here and in most MIMO work [1], [3], is based on a "quasi-static" analysis where the channel varies randomly from burst to burst. Within a burst the channel is assumed fixed and it is also assumed that sufficient bits are transmitted for the standard infinite time horizon of information theory to be meaningful. A second note is that our discussion will concentrate on single user MIMO systems but many results also apply to multiuser systems with receive diversity. Finally, the linear capacity growth is only valid under certain channel conditions. It was originally derived for the independent and identically distributed (i.i.d.) flat Rayleigh fading channel and does not hold true for all cases. For example, if large numbers of antennas are packed into small volumes, then the gains in $H$ may become highly correlated and the linear relationship will plateau out due to the effects of antenna correlation [28]-[30]. In contrast, other propagation effects not captured in (4) may serve to reinforce the capacity gains of MIMO such as multipath delay spread. This was shown in particular in the case when the transmit channel is known [4] but also in the case when it is unknown [5].

More generally, the effect of the channel model is critical. Environments can easily be chosen which give channels where the MIMO capacities do not increase linearly with the numbers of antennas. However, most measurements and models available to date do give rise to channel capacities which are of the same order of magnitude as the promised theory (see Section V). Also the linear growth is usually a reasonable model for moderate numbers of antennas which are not extremely close-packed.

\section{B. Information Theoretic MIMO Capacity}

1) Background: Since feedback is an important component of wireless design (although not a necessary one), it is useful to generalize the capacity discussion to cases that can encompass transmitters having some a priori knowledge of channel. To this end, we now define some central concepts, beginning with the MIMO signal model

$$
\mathbf{r}=\mathbf{H s}+\mathbf{n}
$$

In (5), $\mathbf{r}$ is the $M \times 1$ received signal vector, $\mathbf{s}$ is the $N \times 1$ transmitted signal vector and $\mathbf{n}$ is an $M \times 1$ vector of additive noise terms, assumed i.i.d. complex Gaussian with each element having a variance equal to $\sigma^{2}$. For convenience we normalize the noise power so that $\sigma^{2}=1$ in the remainder of this section. Note that the system equation represents a single MIMO user communicating over a fading channel with additive white Gaussian noise (AWGN). The only interference present is self-interference between the input streams to the MIMO system. Some authors have considered more general systems but most information theoretic results can be discussed in this simple context, so we use (5) as the basic system equation.

Let $\mathbf{Q}$ denote the covariance matrix of $\mathbf{s}$, then the capacity of the system described by (5) is given by [3], [21]

$$
C=\log _{2}\left[\operatorname{det}\left(\mathbf{I}_{M}+\mathbf{H} \mathbf{Q} \mathbf{H}^{*}\right)\right] \quad \mathrm{b} / \mathrm{s} / \mathrm{Hz}
$$

where $\operatorname{tr}(\mathbf{Q}) \leq \rho$ holds to provide a global power constraint. Note that for equal power uncorrelated sources $\mathbf{Q}=(\rho / N) \mathbf{I}_{N}$ and (6) collapses to (4). This is optimal when $\mathbf{H}$ is unknown at the transmitter and the input distribution maximizing the mutual information is the Gaussian distribution [3], [21]. With channel feedback $\mathbf{H}$ may be known at the transmitter and the optimal $\mathbf{Q}$ is not proportional to the identity matrix but is constructed from a waterfilling argument as discussed later.

The form of equation (6) gives rise to two practical questions of key importance. First, what is the effect of $\mathbf{Q}$ ? If we compare the capacity achieved by $\mathbf{Q}=(\rho / N) \mathbf{I}_{N}$ (equal power transmission or no feedback) and the optimal $\mathbf{Q}$ based on perfect channel estimation and feedback, then we can evaluate a maximum capacity gain due to feedback. The second question concerns the effect of the $\mathbf{H}$ matrix. For the i.i.d. Rayleigh fading case we have the impressive linear capacity growth discussed above. For a wider range of channel models including, for example, correlated fading and specular components, we must ask whether this behavior still holds. Below we report a variety of work on the effects of feedback and different channel models.

It is important to note that (4) can be rewritten as [3]

$$
C_{\mathrm{EP}}=\sum_{i=1}^{m} \log _{2}\left(1+\frac{\rho}{N} \lambda_{i}\right) \quad \mathrm{b} / \mathrm{s} / \mathrm{Hz}
$$

where $\lambda_{1}, \lambda_{2}, \ldots, \lambda_{m}$ are the nonzero eigenvalues of $\mathbf{W}, m=$ $\min (M, N)$, and

$$
\mathbf{W}= \begin{cases}\mathbf{H} \mathbf{H}^{*}, & M \leq N \\ \mathbf{H}^{*} \mathbf{H}, & N<M .\end{cases}
$$

This formulation can be easily obtained from the direct use of eigenvalue properties. Alternatively, we can decompose the MIMO channel into $\mathrm{m}$ equivalent parallel SISO channels by performing a singular value decomposition (SVD) of $\mathbf{H}$ [3], [21]. Let the SVD be given by $\mathbf{H}=\mathbf{U D V}^{*}$, then $\mathbf{U}$ and $\mathbf{V}$ are unitary and $\mathbf{D}$ is diagonal with entries specified by $\mathbf{D}=\operatorname{diag}\left(\sqrt{\lambda_{1}}, \sqrt{\lambda_{2}}, \ldots, \sqrt{\lambda_{m}}, 0, \ldots, 0\right)$. Hence (5) can be rewritten as

$$
\tilde{\mathbf{r}}=\mathbf{D} \tilde{\mathbf{s}}+\tilde{\mathbf{n}}
$$

where $\tilde{\mathbf{r}}=\mathbf{U}^{*} \mathbf{r}, \tilde{\mathbf{s}}=\mathbf{V}^{*} \mathbf{s}$ and $\tilde{\mathbf{n}}=\mathbf{U}^{*} \mathbf{n}$. Equation (9) represents the system as $m$ equivalent parallel SISO eigen-channels with signal powers given by the eigenvalues $\lambda_{1}, \lambda_{2}, \ldots, \lambda_{m}$.

Hence, the capacity can be rewritten in terms of the eigenvalues of the sample covariance matrix $\mathbf{W}$. In the i.i.d. Rayleigh fading case, $\mathbf{W}$ is also called a Wishart matrix. Wishart matrices have been studied since the 1920s and a considerable amount is known about them. For general $\mathbf{W}$ matrices a wide range of limiting results are known [22], [31]-[34] as $M$ or $N$ or both tend to infinity. In the particular case of Wishart matrices, many exact results are also available [31], [35]. There is not a great deal of information about intermediate results (neither limiting nor Wishart), but we are helped by the remarkable accuracy of some asymptotic results even for small values of $M, N$ [36].

We now give a brief overview of exact capacity results, broken down into the two main scenarios, where the channel is either known or unknown at the transmitter. We focus on the 
two key questions posed above; what is the effect of feedback and what is the impact of the channel?

2) Channel Known at the Transmitter (Waterfilling): When the channel is known at the transmitter (and at the receiver), then $\mathbf{H}$ is known in (6) and we optimize the capacity over $\mathbf{Q}$ subject to the power constraint $\operatorname{tr}(\mathbf{Q}) \leq \rho$. Fortunately, the optimal $\mathbf{Q}$ in this case is well known [3], [4], [21], [26], [37]-[39] and is called a waterfilling (WF) solution. There is a simple algorithm to find the solution [3], [21], [26], [37], [39] and the resulting capacity is given by

$$
C_{\mathrm{WF}}=\sum_{i=1}^{m} \log _{2}\left(\mu \lambda_{i}\right)^{+} \mathrm{b} / \mathrm{s} / \mathrm{Hz}
$$

where $\mu$ is chosen to satisfy

$$
\rho=\sum_{i=1}^{m}\left(\mu-\lambda_{i}^{-1}\right)^{+}
$$

and "+" denotes taking only those terms which are positive. Since $\mu$ is a complicated nonlinear function of $\lambda_{1}, \lambda_{2}, \ldots, \lambda_{m}$, the distribution of $C_{\mathrm{WF}}$ appears intractable, even in the Wishart case when the joint distribution of $\lambda_{1}, \lambda_{2}, \ldots, \lambda_{m}$ is known. Nevertheless, $C_{\mathrm{WF}}$ can be simulated using (10) and (11) for any given $\mathbf{W}$ so that the optimal capacity can be computed numerically for any channel.

The effect on $C_{\mathrm{WF}}$ of various channel conditions has been studied to a certain extent. For example in Ricean channels increasing the LOS strength at fixed SNR reduces capacity [23], [40]. This can be explained in terms of the channel matrix rank [25] or via various eigenvalue properties. The issue of correlated fading is of considerable importance for implementations where the antennas are required to be closely spaced (see Section VI). Here, certain correlation patterns are being standardized as suitable test cases [41]. A wide range of results in this area is given in [26].

In terms of the impact of feedback (channel information being supplied to the transmitter), it is interesting to note that the WF gains over EP are significant at low SNR but converge to zero as the SNR increases [39], [40], [42]. The gains provided by WF appear to be due to the correlations in $\mathbf{Q}$ rather than any unequal power allocation along the diagonal in $\mathbf{Q}$. This was shown in [40], where the gains due to unequal power uncorrelated sources were shown to be small compared to waterfilling. Over a wide range of antenna numbers and channel models the gains due to feedback are usually less than $30 \%$ for SNR above $10 \mathrm{~dB}$. From zero to $10 \mathrm{~dB}$ the gains are usually less than $60 \%$. For SNR values below $0 \mathrm{~dB}$, large gains are possible, with values around $200 \%$ being reported at $-10 \mathrm{~dB}$. These results are available in the literature, see for example [39], but some simulations are also given in Fig. 3 for completeness. The fact that feedback gain reduces at higher SNR levels can be intuitively explained by the following fact. Knowledge of the transmit channel mainly provides transmit array gain. In contrast, gains such as diversity gain and multiplexing gain do not require this knowledge as these gains can be captured by "blind" transmit schemes such as STCs and V-BLAST (see later). Since the relative importance of transmit array gain in boosting average SNR decreases in the high SNR region, the benefit of feedback also reduces.

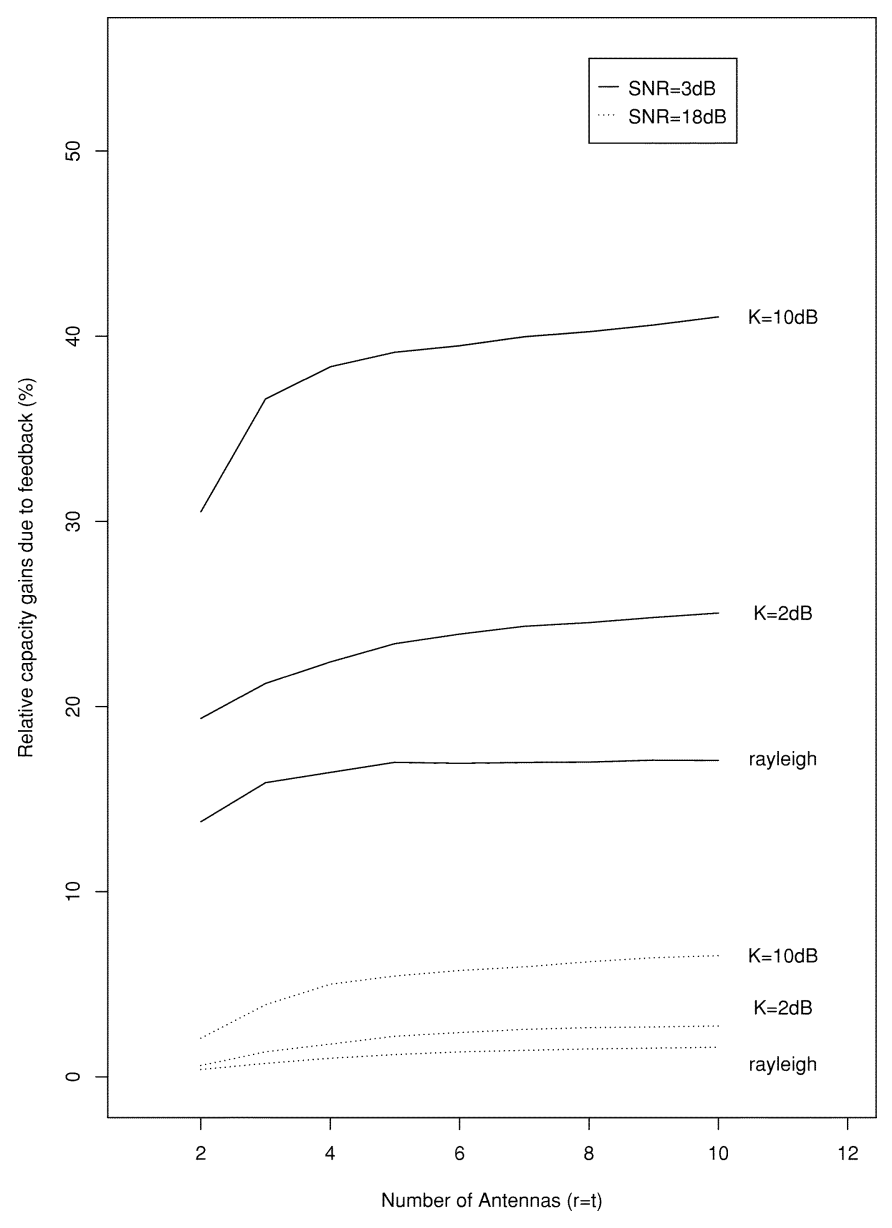

Fig. 3. Shows the percentage relative gains in capacity due to feedback at various SNR values, channel models ( $K$ is the Ricean factor), and array sizes.

3) Channel Unknown at the Transmitter: Here, the capacity is given by $C_{\mathrm{EP}}$ in (4). This was derived by Foschini [1] and Telatar [3], [21] from two viewpoints. Telatar [3], [21] started from (6) and showed that $\mathbf{Q}=(\rho / N) I_{N}$ is optimal for i.i.d. Rayleigh fading. Foschini derived (4) starting from an equal power assumption. The variable, $C_{\mathrm{EP}}$, is considerably more amenable to analysis than $C_{\mathrm{WF}}$. For example, the mean capacity is derived in [3], [21] and the variance in [36] for i.i.d. Rayleigh fading, as well as [43]. In addition, the full moment generating function (MGF) for $C_{\mathrm{EP}}$ is given in [27] although this is rather complicated being in determinant form. Similar results include [44].

For more complex channels, results are rapidly becoming available. Again, capacity is reduced in Ricean channels as the relative LOS strength increases [25], [37] . The impact of correlation is important and various physical models and measurements of correlations have been used to assess its impact [26], [45]-[47]. For example, $C_{\mathrm{EP}}$ is shown to plateau out as the number of antennas increases in either sparse scattering environments [48] or dense/compact MIMO arrays [29], [30].

\section{Limiting Capacity Results}

The exact results of Section III-B above are virtually all dependent on the i.i.d. Rayleigh fading (Wishart) case. For other scenarios exact results are few and far between. Hence, it is 
useful to pursue limiting results not only to cover a broader range of cases but also to give simpler and more intuitive results and to study the potential of very large scale systems. The surprising thing about limiting capacity results is their accuracy. Many authors have considered the limiting case where $M, N \rightarrow \infty$ and $M / N \rightarrow c$ for some constant $c$. This represents the useful case where the number of antennas grow proportionally at both TX and RX. Limiting results in this sense we denote as holding for "large systems." In particular, it covers the most interesting special case where $M=N$ and both become large. It turns out that results based on this limiting approach are useful approximations even down to $M=2$ ! [36], [40], [49], [50]. We outline this work below, as well as results which are asymptotic in SNR rather than system size.

1) Channel Known at the Transmitter: Analytical results are scarce here but a nice analysis in [39] and [42] shows that $C_{\mathrm{WF}} / M$ converges to a constant, $\mu_{\mathrm{WF}}$, for "large systems" in both i.i.d. and correlated fading conditions. The value of $\mu_{\mathrm{WF}}$ is given by an integral equation. The rest of our "large system" knowledge is mainly based on simulations. For example, linear growth of $C_{\mathrm{WF}}$ is shown for Ricean fading in [40] as is the accuracy of Gaussian approximations to $C_{\mathrm{WF}}$ in both Rayleigh and Ricean cases.

In terms of SNR asymptotics for "large systems," [39] gives both low and high SNR results.

2) Channel Unknown at the Transmitter: In this situation, we have the capacity given in (4) as $C_{\mathrm{EP}}$. For "large systems" (assuming the Wishart case) the limiting mean capacity was shown to be of the form $M \mu_{\mathrm{EP}}$ [3] where $\mu_{\mathrm{EP}}$ depends on $M$, $N$ only through the ratio $c=M / N$. A closed form expression for $C_{\mathrm{EP}}$ was given in [23] and the accuracy of this result was demonstrated in [36] and [40]. The limiting variance is a constant [27], again dependent on $c$ rather than $M$ and $N$ individually. Convergence rates to this constant are indicated in [36] [40]. In fact, for a more general class of fading channels similar results hold and a central limit theorem can be stated [33], [34] as below

$$
\lim _{M, N \rightarrow \infty}\left(\frac{C_{\mathrm{EP}}-E\left(C_{\mathrm{EP}}\right)}{\sqrt{\operatorname{Var}\left(C_{\mathrm{EP}}\right)}}\right)=Z
$$

where $M / N \rightarrow c$ as $M, N \rightarrow \infty$ and $Z \sim N(0,1)$ is a standard Gaussian random variable. See [33] and [34] for exact details of the conditions required for (12) to hold. Hence, for the Wishart case Gaussian approximations might be considered to $C_{\mathrm{EP}}$ using the exact mean and variance [3], [21], [36] or limiting values [23], [27]. These have been shown to be surprisingly accurate, even down to $M=2$ [36], [40], not only for Rayleigh channels, but for Ricean channels as well. More general results which also cater for correlated fading can be found in [27], [39], and [42]. In [39] and [42], it is shown that $C_{\mathrm{EP}} / M$ converges to a constant, $\mu_{\mathrm{EP}}$, for "large systems" in both i.i.d. and correlated fading. The value of $\mu_{\mathrm{EP}}$ is obtained and it is shown that correlation always reduces $\mu_{\mathrm{EP}}$. In [27], a powerful technique is used to derive limiting results for the mean and variance in both i.i.d. and correlated fading.

Moving onto results which are asymptotic in SNR, [39] gives both low and high SNR capacity results for "large systems." It is shown that at high $\mathrm{SNR}, C_{\mathrm{EP}}$, and $C_{\mathrm{WF}}$ are equivalent. For arbitrary values of $M, N$ high SNR approximations are given in [27] for the mean, variance, and MGF of $C_{\mathrm{EP}}$.

\section{TRAnSmission OVER MIMO Systems}

Although the information theoretic analysis can be bootstrapped to motivate receiver architectures (as was done, e.g., in [1], [2]), it usually carries a pitfall in that it does not reflect the performance achieved by actual transmission systems, since it only provides an upper bound realized by algorithms/codes with boundless complexity or latency. The development of algorithms with a reasonable BER performance/complexity compromise is required to realize the MIMO gains in practice. Here, we summarize different MIMO transmission schemes, give the intuition behind them, and compare their performance.

\section{A. General Principles}

Current transmission schemes over MIMO channels typically fall into two categories: data rate maximization or diversity maximization schemes, although there has been some effort toward unification recently. The first kind focuses on improving the average capacity behavior. For example, in the example shown in Fig. 2, the objective is just to perform spatial multiplexing as we send as many independent signals as we have antennas for a specific error rate (or a specific outage capacity [2]).

More generally, however, the individual streams should be encoded jointly in order to protect transmission against errors caused by channel fading and noise plus interference. This leads to a second kind of approach in which one tries also to minimize the outage probability, or equivalently maximize the outage capacity.

Note that if the level of redundancy is increased between the TX antennas through joint coding, the amount of independence between the signals decreases. Ultimately, it is possible to code the signals so that the effective data rate is back to that of a single antenna system. Effectively, each TX antenna then sees a differently encoded, fully redundant version of the same signal. In this case, the multiple antennas are only used as a source of spatial diversity and not to increase data rate, or at least not in a direct manner.

The set of schemes aimed at realizing joint encoding of multiple TX antennas are called STCs. In these schemes, a number of code symbols equal to the number of TX antennas are generated and transmitted simultaneously, one symbol from each antenna. These symbols are generated by the space-time encoder such that by using the appropriate signal processing and decoding procedure at the receiver, the diversity gain and/or the coding gain is maximized. Fig. 4 shows a simple block diagram for STC.

The first attempt to develop STC was presented in [51] and was inspired by the delay diversity scheme of Wittneben [52]. However, the key development of the STC concept was originally revealed in [53] in the form of trellis codes, which required a multidimensional (vector) Viterbi algorithm at the receiver for decoding. These codes were shown to provide a diversity benefit equal to the number of TX antennas in addition to a coding gain that depends on the complexity of the code (i.e., number of states in the trellis) without any loss in bandwidth efficiency. 


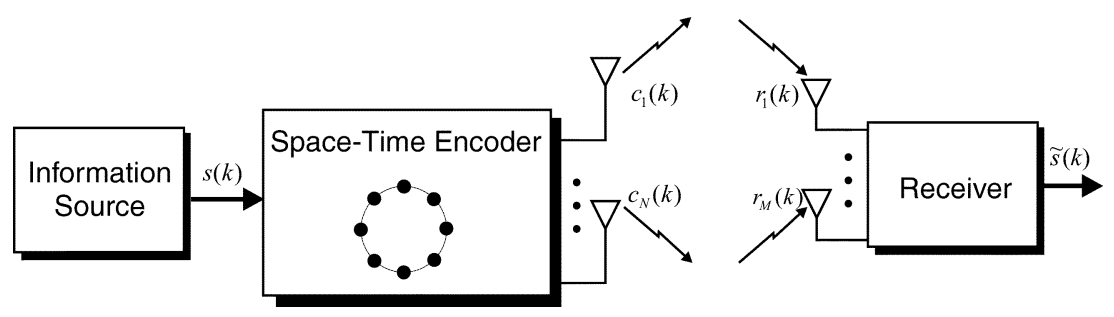

Fig. 4. Space-time coding.

Then, the popularity of STC really took off with the discovery of the so-called space-time block codes (STBCs). This is due to the fact that because of their construction, STBC can be decoded using simple linear processing at the receiver [in contrast to the vector Viterbi required for ST trellis codes (STTC)]. Although STBC codes give the same diversity gain as the STTC for the same number of TX antennas, they provide zero or minimal coding gain. Below, we will briefly summarize the basic concepts of STC and then extensions to the case of multiple RX antennas (MIMO case). As the reader will note, emphasis within space-time coding is placed on block approaches, which seem to currently dominate the literature rather than on trellis-based approaches. A more detailed summary of Sections IV-B and IV-C can be found in [54].

\section{B. Maximizing Diversity With STTC}

For every input symbol $s_{l}$, a space-time encoder generates $N$ code symbols $c_{l 1}, c_{l 2}, \ldots, c_{l N}$. These $N$ code symbols are transmitted simultaneously from the $N$ transmit antennas. We define the code vector as $\mathbf{c}_{l}=\left[\begin{array}{llll}c_{l 1} & c_{l 2} & \cdots & c_{l N}\end{array}\right]^{T}$. Suppose that the code vector sequence

$$
\mathbf{C}=\left\{\mathbf{c}_{1}, \mathbf{c}_{2}, \ldots, \mathbf{c}_{L}\right\}
$$

was transmitted. We consider the probability that the decoder decides erroneously in favor of the legitimate code vector sequence

$$
\tilde{\mathbf{C}}=\left\{\tilde{\mathbf{c}}_{1}, \tilde{\mathbf{c}}_{2}, \ldots, \tilde{\mathbf{c}}_{L}\right\}
$$

Consider a frame or block of data of length $L$ and define the $N \times N$ error matrix $\mathbf{A}$ as

$$
\mathbf{A}(\mathbf{C}, \tilde{\mathbf{C}})=\sum_{l=1}^{L}\left(\mathbf{c}_{l}-\tilde{\mathbf{c}}_{l}\right)\left(\mathbf{c}_{l}-\tilde{\mathbf{c}}_{l}\right)^{*} .
$$

If ideal channel state information (CSI) $\mathbf{H}(l), l=1, \ldots, L$ is available at the receiver, then it is possible to show that the probability of transmitting $\mathbf{C}$ and deciding in favor of $\tilde{\mathbf{C}}$ is upper bounded for a Rayleigh fading channel by [20]

$$
P(\mathbf{C} \rightarrow \tilde{\mathbf{C}}) \leq\left(\prod_{i=1}^{r} \beta_{i}\right)^{-M} \cdot\left(E_{s} / 4 N_{o}\right)^{-r M}
$$

where $E_{s}$ is the symbol energy and $N_{o}$ is the noise spectral density, $r$ is the rank of the error matrix $\mathbf{A}$ and $\beta_{i}, i=1, \ldots, r$ are the nonzero eigenvalues of the error matrix $\mathbf{A}$. We can easily see that the probability of error bound in (14) is similar to the probability of error bound for trellis coded modulation for fading channels. The term $g_{r}=\prod_{i=1}^{r} \beta_{i}$ represents the coding gain achieved by the STC and the term $\left(E_{s} / 4 N_{o}\right)^{-r M}$ represents a
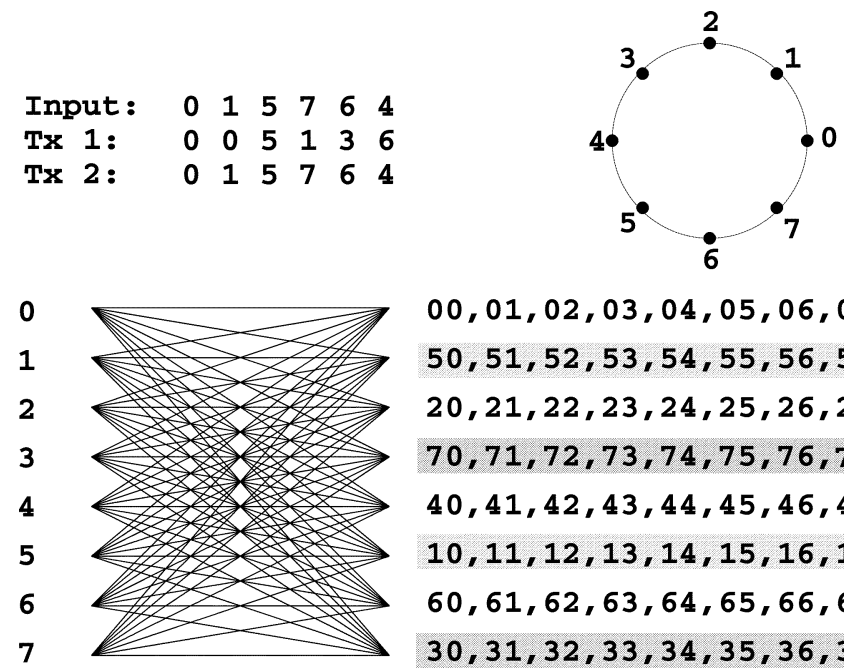

$00,01,02,03,04,05,06,07$

$50,51,52,53,54,55,56,57$

$20,21,22,23,24,25,26,27$

$70,71,72,73,74,75,76,77$

$40,41,42,43,44,45,46,47$

$10,11,12,13,14,15,16,17$

$60,61,62,63,64,65,66,67$

$30,31,32,33,34,35,36,37$

\section{8-PSK 8-State Space-Time Code with 2 Tx Antennas}

Fig. 5. The 8-PSK 8-state STC with two TX antennas.

diversity gain of $r M$. Since $r \leq N$, the overall diversity order is always less or equal to $M N$. It is clear that in designing a STTC, the rank of the error matrix $r$ should be maximized (thereby maximizing the diversity gain) and at the same time $g_{r}$ should also be maximized, thereby maximizing the coding gain.

As an example for STTCs, we provide an 8-PSK eight-state STC designed for two TX antennas. Fig. 5 provides a labeling of the 8-PSK constellation and the trellis description for this code. Each row in the matrix shown in Fig. 5 represents the edge labels for transitions from the corresponding state. The edge label $s_{1} s_{2}$ indicates that symbol $s_{1}$ is transmitted over the first antenna and that symbol $s_{2}$ is transmitted over the second antenna. The input bit stream to the ST encoder is divided into groups of 3 bits and each group is mapped into one of eight constellation points. This code has a bandwidth efficiency of 3 bits per channel use.

Fig. 6 shows the performance of 4-PSK STTCs for two TX and one RX antennas with different number of states.

Since the original STTC were introduced by Tarokh et al. in [53], there has been extensive research aiming at improving the performance of the original STTC designs. These original STTC designs were hand crafted (according to the proposed design criteria) and, therefore, are not optimum designs. In recent years, a large number of research proposals have been published which propose new code constructions or perform systematic searches for different convolutional STTC or some variant of the original design criteria proposed by Tarokh et al. Examples of such work can be found in [55]-[60] (these are mentioned only as an example, there are many other published results that address 


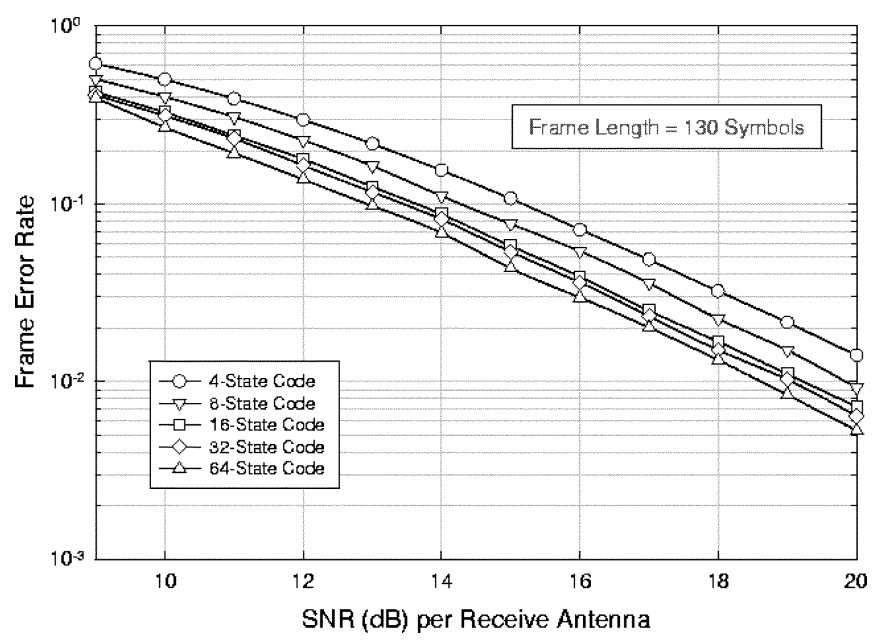

Fig. 6. Performance of 4-PSK STTCs with two TX and one RX antennas.

the same issue, too numerous to list here). These new code constructions provide an improved coding advantage over the original scheme by Tarokh et al., however, only marginal gains were obtained in most cases.

\section{Maximizing Diversity With STBCs}

When the number of antennas is fixed, the decoding complexity of space-time trellis coding (measured by the number of trellis states at the decoder) increases exponentially as a function of the diversity level and transmission rate [53]. In addressing the issue of decoding complexity, Alamouti [61] discovered a remarkable space-time block coding scheme for transmission with two antennas. This scheme supports maximum-likelihood (ML) detection based only on linear processing at the receiver. The very simple structure and linear processing of the Alamouti construction makes it a very attractive scheme that is currently part of both the W-CDMA and CDMA-2000 standards. This scheme was later generalized in [62] to an arbitrary number of antennas. Here, we will briefly review the basics of STBCs. Fig. 7 shows the baseband representation for Alamouti STBC with two antennas at the transmitter. The input symbols to the space-time block encoder are divided into groups of two symbols each. At a given symbol period, the two symbols in each group $\left\{c_{1}, c_{2}\right\}$ are transmitted simultaneously from the two antennas. The signal transmitted from antenna 1 is $c_{1}$ and the signal transmitted from antenna 2 is $c_{2}$. In the next symbol period, the signal $-c_{2}^{*}$ is transmitted from antenna 1 and the signal $c_{1}^{*}$ is transmitted from antenna 2 . Let $h_{1}$ and $h_{2}$ be the channels from the first and second TX antennas to the RX antenna, respectively. The major assumption here is that $h_{1}$ and $h_{2}$ are scalar and constant over two consecutive symbol periods, that is

$$
h_{i}(2 n T) \approx h_{i}((2 n+1) T), \quad i=1,2 .
$$

We assume a receiver with a single RX antenna. we also denote the received signal over two consecutive symbol periods as $r_{1}$ and $r_{2}$. The received signals can be expressed as

$$
\begin{aligned}
& r_{1}=h_{1} c_{1}+h_{2} c_{2}+n_{1} \\
& r_{2}=-h_{1} c_{2}^{*}+h_{2} c_{1}^{*}+n_{2}
\end{aligned}
$$

where $n_{1}$ and $n_{2}$ represent the AWGN and are modeled as i.i.d. complex Gaussian random variables with zero mean and power spectral density $N_{o} / 2$ per dimension. We define the received signal vector $\mathbf{r}=\left[\begin{array}{ll}r_{1} & r_{2}^{*}\end{array}\right]^{T}$, the code symbol vector $\mathbf{c}=\left[\begin{array}{ll}c_{1} & c_{2}\end{array}\right]^{T}$, and the noise vector $\mathbf{n}=\left[\begin{array}{ll}n_{1} & n_{2}^{*}\end{array}\right]^{T}$. Equations (15) and (16) can be rewritten in a matrix form as

$$
\mathbf{r}=\mathbf{H} \cdot \mathbf{c}+\mathbf{n}
$$

where the channel matrix $\mathbf{H}$ is defined as

$$
\mathbf{H}=\left[\begin{array}{rr}
h_{1} & h_{2} \\
h_{2}^{*} & -h_{1}^{*}
\end{array}\right] .
$$

$\mathbf{H}$ is now only a virtual MIMO matrix with space (columns) and time (rows) dimensions, not to be confused with the purely spatial MIMO channel matrix defined in previous sections. The vector $\mathbf{n}$ is a complex Gaussian random vector with zero mean and covariance $N_{o} \cdot \mathbf{I}_{2}$. Let us define $\mathbf{C}$ as the set of all possible symbol pairs $\mathbf{c}=\left\{c_{1}, c_{2}\right\}$. Assuming that all symbol pairs are equiprobable, and since the noise vector $\mathbf{n}$ is assumed to be a multivariate AWGN, we can easily see that the optimum ML decoder is

$$
\hat{\mathbf{c}}=\arg \min _{\hat{\mathbf{c}} \in \mathbf{C}}\|\mathbf{r}-\mathbf{H} \cdot \hat{\mathbf{c}}\|^{2} .
$$

The ML decoding rule in (19) can be further simplified by realizing that the channel matrix $\mathbf{H}$ is always orthogonal regardless of the channel coefficients. Hence, $\mathbf{H}^{*} \mathbf{H}=\alpha \cdot \mathbf{I}_{2}$ where $\alpha=\left|h_{1}\right|^{2}+\left|h_{2}\right|^{2}$. Consider the modified signal vector $\tilde{\mathbf{r}}$ given by

$$
\tilde{\mathbf{r}}=\mathbf{H}^{*} \cdot \mathbf{r}=\alpha \cdot \mathbf{c}+\tilde{\mathbf{n}}
$$

where $\tilde{\mathbf{n}}=\mathbf{H}^{*} \cdot \mathbf{n}$. In this case, the decoding rule becomes

$$
\hat{\mathbf{c}}=\arg \min _{\hat{\mathbf{c}} \in \mathbf{C}}\|\tilde{\mathbf{r}}-\alpha \cdot \hat{\mathbf{c}}\|^{2} .
$$

Since $\mathbf{H}$ is orthogonal, we can easily verify that the noise vector $\tilde{\mathbf{n}}$ will have a zero mean and covariance $\alpha N_{o} \cdot \mathbf{I}_{2}$, i.e., the elements of $\tilde{\mathbf{n}}$ are i.i.d. Hence, it follows immediately that by using this simple linear combining, the decoding rule in (21) reduces to two separate, and much simpler decoding rules for $c_{1}$ and $c_{2}$, as established in [61]. In fact, for the above $2 \times 1 \mathrm{STBC}$, only two complex multiplications and one complex addition per symbol are required for decoding. Also, assuming that we are using a signaling constellation with $2^{b}$ constellation points, this linear combining reduces the number of decoding metrics that has to be computed for ML decoding from $2^{2 b}$ to $2 \times 2^{b}$. It is also straightforward to verify that the SNR for $c_{1}$ and $c_{2}$ will be

$$
\mathrm{SNR}=\frac{\alpha \cdot E_{s}}{N_{o}}
$$

and, hence, a two branch diversity performance (i.e., a diversity gain of order two) is obtained at the receiver.

MIMO Extensions: Initially developed to provide transmit diversity in the MISO case, STCs are readily extended to the MIMO case. When the receiver uses $M \mathrm{RX}$ antennas, the received signal vector $\mathbf{r}_{\mathrm{m}}$ at $\mathrm{RX}$ antenna $m$ is

$$
\mathbf{r}_{m}=\mathbf{H}_{m} \cdot \mathbf{c}+\mathbf{n}_{m}
$$




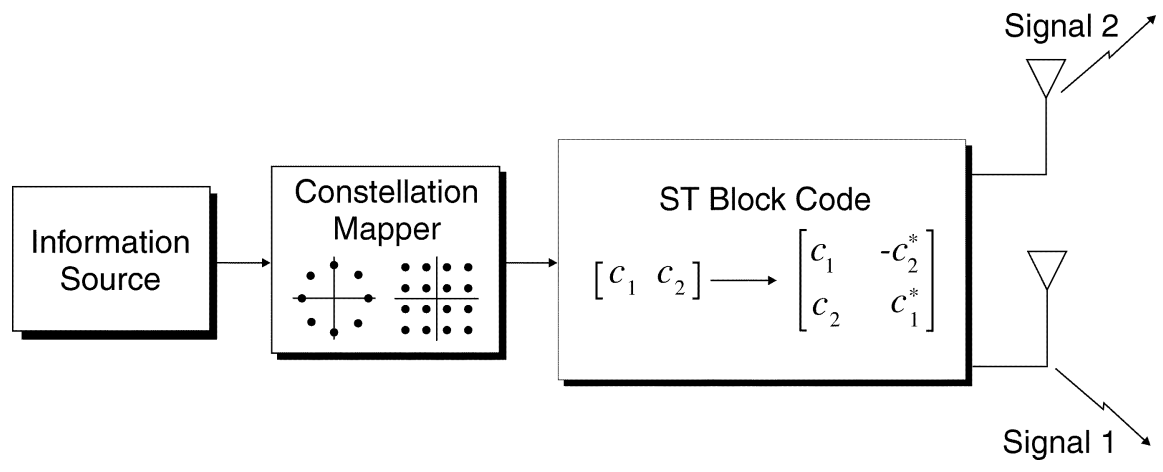

Fig. 7. Transmitter diversity with space-time block coding.

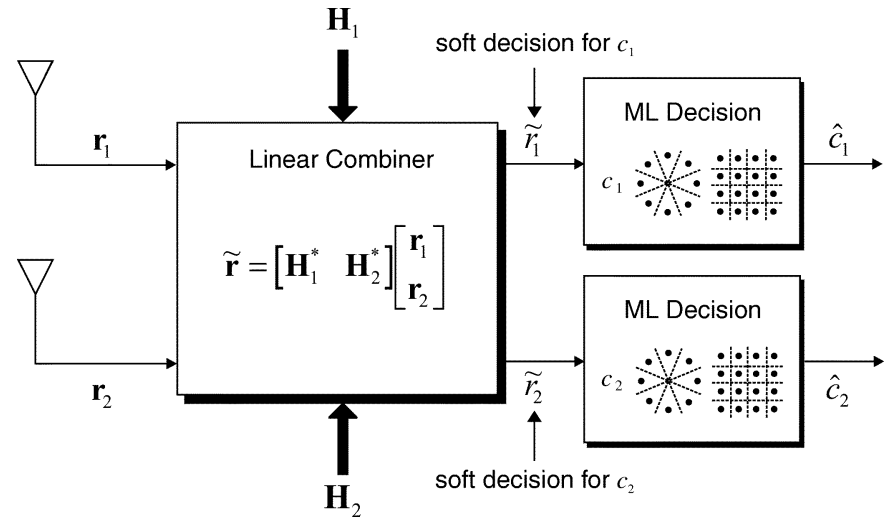

Fig. 8. Receiver for space-time block coding.

where $\mathbf{n}_{m}$ is the noise vector at the two time instants and $\mathbf{H}_{m}$ is the channel matrix from the two TX antennas to the $m$ th receive antenna. In this case, the optimum ML decoding rule is

$$
\hat{\mathbf{c}}=\arg \min _{\hat{\mathbf{c}} \in \mathbf{C}} \sum_{m=1}^{M}\left\|\mathbf{r}_{m}-\mathbf{H}_{m} \cdot \hat{\mathbf{c}}\right\|^{2} .
$$

As before, in the case of $M \mathrm{RX}$ antennas, the decoding rule can be further simplified by premultiplying the received signal vector $\mathbf{r}_{m}$ by $\mathbf{H}_{m}^{*}$. In this case, the diversity order provided by this scheme is $2 M$. Fig. 8 shows a simplified block diagram for the receiver with two RX antennas. Note that the decision rule in (21) and (24) amounts to performing a hard decision on $\tilde{\mathbf{r}}$ and $\tilde{\mathbf{r}}_{M}=\sum_{m=1}^{M} \mathbf{H}_{m}^{*} \mathbf{r}_{m}$, respectively. Therefore, as shown in Fig. 8, the received vector after linear combining, $\tilde{\mathbf{r}}_{M}$, can be considered as a soft decision for $c_{1}$ and $c_{2}$. Hence, in the case the STBC is concatenated with an outer conventional channel code, like a convolutional code, these soft decisions can be fed to the outer channel decoder to yield a better performance. Note also that for the above $2 \times 2 \mathrm{STBC}$, the transmission rate is one while achieving the maximum diversity gain possible with two TX and two RX antennas (fourth order). However, concatenating a STBC with an outer conventional channel code (e.g., a convolutional or trellis coded modulation (TCM) code) will incur a rate loss. A very clever method to concatenate STBC based on the Alamouti scheme with an outer TCM or convolutional code was originally presented in [63]-[65]. In this approach, the cardinality of the inner STBC is enlarged to form an expanded orthogonal space-time signal set or constellation. This set is obtained by applying a unitary transformation to the original Alamouti scheme. Once this expanded space-time signal constellation is formed, the design of a good space-time TCM code based on this signal set is pretty much analogous to classic TCM code design. In other words, classic set partitioning techniques are used to partition signals within each block code subset. Thus, a combined STBC-TCM construct is generated and guaranteed to achieve full diversity by using a simple design rule that restricts the transition branches leaving from or arriving to each state to be labeled by codewords from the same block code subset. This rule is the same as the original design rule of STTC proposed by Tarokh et al. in [53]. A similar scheme was later presented in [66]. The extension of the above STBC to more than two TX antennas was studied in [62] and [67]-[69]. There, a general technique for constructing STBCs for $N>2$ that provide the maximum diversity promised by the number of TX and RX antennas was developed. These codes retain the simple ML decoding algorithm based on only linear processing at the receiver [61]. It was also shown that for real signal constellations, i.e., PAM constellation, STBCs with transmission rate 1 can be constructed [62]. However, for general complex constellations like M-QAM or M-PSK, it is not known whether a STBC with transmission rate 1 and simple linear processing that will give the maximum diversity gain with $N>2 \mathrm{TX}$ antennas does exist or not. Moreover, it was also shown that such a code where the number of TX antennas $N$ equals the number of both the number of information symbols transmitted and the number of time slots needed to transmit the code block does not exist. However, for rates $<1$, such codes can be found. For example, assuming that the transmitter unit uses four TX antennas, a rate $4 / 8$ (i.e., it is a rate $1 / 2$ ) STBC is given by

$$
C=\left[\begin{array}{rrrrrrrr}
c_{1} & -c_{2} & -c_{3} & -c_{4} & c_{1}^{*} & -c_{2}^{*} & -c_{3}^{*} & -c_{4}^{*} \\
c_{2} & c_{1} & c_{4} & -c_{3} & c_{2}^{*} & c_{1}^{*} & c_{4}^{*} & -c_{3}^{*} \\
c_{3} & -c_{4} & c_{1} & c_{2} & c_{3}^{*} & -c_{4}^{*} & c_{1}^{*} & c_{2}^{*} \\
c_{4} & c_{3} & -c_{2} & c_{1} & c_{4}^{*} & c_{3}^{*} & -c_{2}^{*} & c_{1}^{*}
\end{array}\right] .
$$

In this case, at time $t=1, c_{1}, c_{2}, c_{3}, c_{4}$ are transmitted from antenna 1 through 4 , respectively. At time $t=2,-c_{2}, c_{1},-c_{4}, c_{3}$ are transmitted from antenna 1 through 4 , respectively, and so on. For this example, rewriting the received signal in a way analogous to (17) (where $\mathbf{c}=\left[c_{1}, \ldots, c_{4}\right]$ ) will yield a $8 \times 4$ virtual MIMO matrix $\mathbf{H}$ that is orthogonal i.e., the decoding is linear, and $\mathbf{H}^{*} \mathbf{H}=\alpha_{4} \cdot \mathbf{I}$, where $\alpha_{4}=2 \cdot \sum_{i=1}^{4}\left|h_{i}\right|^{2}$ (fourth-order diversity). This scheme provides a $3-\mathrm{dB}$ power gain that comes 
from the intuitive fact that eight time slots are used to transmit four information symbols. The power gain compensates for the rate loss.

As an alternative to the schemes above sacrificing code rate for orthogonality, it is possible to sacrifice orthogonality in an effort to maintain full rate one codes for $N>2$. Quasi-orthogonal STBC were investigated for instance in [70] in which we can preserve the full diversity and full rate at the cost of a small loss in BER performance and some extra decoding complexity relative to truly orthogonal schemes.

$R X$ Channel Knowledge (or Lack of): The decoding of ST block codes above requires knowledge of the channel at the receiver. The channel state information can be obtained at the receiver by sending training or pilot symbols or sequences to estimate the channel from each of the TX antennas to the receive antenna [71]-[78]. For one TX antenna, there exist differential detection schemes, such as differential phase-shift keying (DPSK), that neither require the knowledge of the channel nor employ pilot or training symbol transmission. These differential decoding schemes are used, for example, in the IS-54 cellular standard ( $\pi / 4$-DPSK). This motivates the generalization of differential detection schemes for the case of multiple TX antennas. A partial solution to this problem was proposed in [79] for the $2 \times 2$ code, where it was assumed that the channel is not known at the receiver. In this scheme, the detected pair of symbols at time $t-1$ are used to estimate the channel at the receiver and these channel estimates are used for detecting the pair of symbols at time $t$. However, the scheme in requires the transmission of known pilot symbols at the beginning and, hence, are not fully differential. The scheme in [79] can be thought as a joint data channel estimation approach which can lead to error propagation. In [80], a true differential detection scheme for the $2 \times 2$ code was constructed. This scheme shares many of the desirable properties of DPSK: it can be demodulated with or without CSI at the receiver, achieve full diversity gain in both cases, and there exists a simple noncoherent receiver that performs within $3 \mathrm{~dB}$ of the coherent receiver. However, this scheme has some limitations. First, the encoding scheme expands the signal constellation for nonbinary signals. Second, it is limited only to the $N=2 \mathrm{STBC}$ for a complex constellation and to the case $N \leq 8$ for a real constellation. This is based on the results in [62] that the $2 \times 2$ STBC is an orthogonal design and complex orthogonal designs do not exist for $N>2$. In [81], another approach for differential modulation with transmit diversity based on group codes was proposed. This approach can be applied to any number of antennas and any constellation. The group structure of theses codes greatly simplifies the analysis of these schemes, and may also yield simpler and more transparent modulation and demodulation procedures. A different nondifferential approach to transmit diversity when the channel is not known at the receiver is reported in [82] and [83], but this approach requires exponential encoding and decoding complexities. Additional generalizations on differential STC schemes are given in [84].

\section{STC in Frequency Selective Channels}

Both STTC and STBC codes were first designed assuming a narrowband wireless system, i.e., a flat fading channel.
However, when used over frequency selective channels a channel equalizer has to be used at the receiver along with the space-time decoder. Using classical equalization methods with space-time coded signals is a difficult problem. For example, for STTC designed for two TX antennas and a receiver with one $\mathrm{RX}$ antenna, we need to design an equalizer that will equalize two independent channels (one for each TX antenna) from one receive signal. For the case of the STBC, the nonlinear and noncausal nature of the code makes the use of classical equalization methods [such as the minimum mean square error (MMSE) linear equalizer, decision feedback equalizer (DFE), and maximum-likelihood sequence estimation (MLSE)] a challenging problem.

Initial attempts to address the problem for STTC made use of whatever structure was available in the space-time coded signal [85]-[87], where the structure of the code was used to convert the problem into one that can be solved using known equalization schemes. For the STBC, the equalization problem was addressed by modifying the original Alamouti scheme in such a way that the use over frequency selective channels, and hence the equalization, is a much easier task. For example, in [88], STBC was used in conjunction with OFDM. OFDM is used to convert the frequency selective channel into a set of independent parallel frequency-flat subchannels. The Alamouti scheme is then applied to two consecutive subcarriers (or two consecutive OFDM block). Note that more general code designs can be used [89].

In [90], the Alamouti scheme is imposed on a block basis (not on symbol basis as in the original scheme) and cyclic prefixes are added to each block. Using fast Fourier transform (FFT), a frequency-domain single carrier is used to equalize the channel. This is similar to OFDM except that it is a single carrier transmission system and the decisions are done in the time domain. A similar approach was proposed in [91], where the Alamouti scheme is imposed on block basis in the time domain and guard bands are added. The equalization is achieved by a clever combination of time domain filtering, conjugation, time reversal, and a SISO MLSE equalizer. This scheme is similar to that in [90] except that the equalization is now done in the time domain.

\section{E. Maximizing Data Rate Using Spatial Multiplexing}

Spatial multiplexing, of which V-BLAST [2], [9] is a particular implementation approach, can be regarded as a special class of STBCs where streams of independent data are transmitted over different antennas, thus maximizing the average data rate over the MIMO system. One may generalize the example given in Section II in the following way: Assuming a block of independent data $\mathbf{C}$ of size $N \times L$ is transmitted over the $N \times M$ MIMO system, the receiver will obtain $\mathbf{Y}=\mathbf{H C}+\mathbf{N}$ where $\mathbf{Y}$ is of size $M \times L$. In order to perform symbol detection, the receiver must unmix the channel, in one of several various possible ways. Zero-forcing (ZF) techniques use a straight matrix inversion, a simple approach which can also result in poor results when the matrix $\mathbf{H}$ becomes very ill conditioned as in certain random fading events or in the presence of LOS (see Section V). The use of a MMSE linear receiver may help in this case, but improvements are found to be limited ( 1.5 to $2 \mathrm{~dB}$ in the $2 \times 2$ case) if knowledge of nontrivial noise/interference statistics (e.g., covariance matrix) are not exploited in the MMSE. 
The optimum decoding method on the other hand is ML where the receiver compares all possible combinations of symbols which could have been transmitted with what is observed

$$
\hat{\mathbf{C}}=\arg \min _{\hat{\mathbf{C}}}\|\mathbf{Y}-\mathbf{H} \hat{\mathbf{C}}\| .
$$

The complexity of ML decoding is high, and even prohibitive when many antennas or high-order modulations are used. Enhanced variants of this like sphere decoding [92] have recently been proposed. Another popular decoding strategy proposed along side V-BLAST is known as nulling and canceling which gives a reasonable tradeoff between complexity and performance. The matrix inversion process in nulling and canceling is performed in layers where one estimates a row from $\mathbf{C}$, subtracts the symbol estimates from $\mathbf{Y}$, and continues the decoding successively [9]. Full details and analysis on this approach are provided in [14]. Note that the iterative nulling and canceling approach is reminiscent of the successive interference canceling (SIC) proposed for multiuser detection (MUD) in CDMA receivers [93]. In fact, any proposed MUD algorithm can be recast in the MIMO context if the input of the MIMO system are seen as virtual users. A difference here is that the separation is carried out in the spatial channel domain rather than the code domain, making its success dependent on channel realizations. On the other hand, the complexity of CDMA-SIC is much higher than in the MIMO case since the number of CDMA users may go well beyond the number of virtual users/antennas in a single MIMO link.

Blind Detection: When the channel is not known at the receiver (as well as at the transmitter) the joint detection of MIMO signals must resort to so-called "blind" approaches. Surprisingly, one may note that progress in this area has been initiated long before the results of [1]-[3], in the more general context of blind source separation (see for instance [94]). In these blind array processing techniques, the input sources are mixed linearly by a mixing matrix (here corresponding to the MIMO channel) and separated by exploiting higher order statistics of the receive array signals [95], [96], or covariance subspace estimation [97] and/or some alphabet (modulation format related) information [98] to cite just a few of the many contributions there. The price paid for avoiding channel training in blind approaches is in some limited loss of BER performance and more often in the increased computational complexity.

1) Multiplexing Versus Diversity: Pure spatial multiplexing allows for full independent usage of the antennas, however, it gives limited diversity benefit and is rarely the best transmission scheme for a given BER target. Coding the symbols within a block can result in additional coding and diversity gain which can help improve the performance, even though the data rate is kept at the same level. It is also possible to sacrifice some data rate for more diversity. In turn, the improved BER performance will buy more data rate indirectly through allowing higher level modulations, such as $16 \mathrm{QAM}$ instead of $4 \mathrm{PSK}$, etc. The various tradeoffs between multiplexing and diversity have begun to be looked at, for instance in [99] and [100].

Methods to design such codes start from a general structure where one often assumes that a weighted linear combination of symbols may be transmitted from any given antenna at any given time. The weights themselves are selected in different fashions

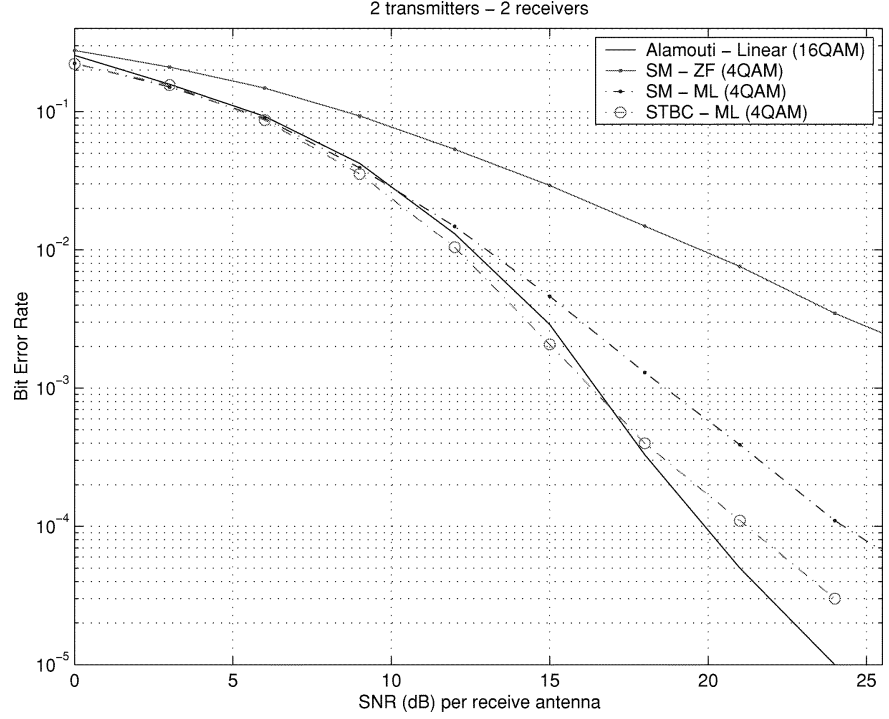

Fig. 9. BER comparisons for various transmission techniques over $2 \times 2$ MIMO. At high SNR, from top to bottom: Spatial multiplexing (SM)-ZF, SM-ML, STBC-ML, Alamouti STBC.

by using analytical tools or optimizing various cost functions [67], [101]-[103].

Spatial multiplexing and space-time block coding can be combined to give a transmission scheme that will maximize the average data rate over the MIMO channel and guarantee a minimum order of diversity benefit for each substream. In fact, the structure of the STBC can be exploited in a way such that the process of detecting and decoding successive steams or layers is a completely linear process. See [54] for more details.

Numerical Comparisons: In what follows, we compare four transmission strategies over a $2 \times 2$ MIMO system with ideally uncorrelated elements. All schemes result in the same nominal rate but offer different BER performance.

Fig. 9 plots the performance of the Alamouti code presented in Fig. 7, spatial multiplexing (SM) with ZF and with ML detection, and a spatial multiplexing scheme with ML decoding using precoding [103]. A 4-QAM constellation is used for the symbols except for the Alamouti code which is simulated under 16 QAM to keep the data rate at the same level as in the other schemes. It can be seen from the figure that spatial multiplexing with ZF returns rather poor results, while the curves for other coding-based methods are quite similar to each other. This is because using two independent streams and a ZF receiver in the $2 \times 2$ case leaves each substream starving for diversity. The Alamouti curve has the best slope at high SNR because it focuses entirely on diversity (order four). At lower SNR, the scheme combining spatial multiplexing with some block coding is the best one because ML decoding allows extraction of some diversity gain in addition to the rate (multiplexing) gain. Note that this benefit comes at the price of receiver complexity compared with Alamouti. In Section VI, we give more comparisons with system-based constraints.

It is important to note that as the number of antennas increases, the diversity effect will give diminishing returns. In contrast, the data rate gain of spatial multiplexing remains linear with the number of antennas. Therefore, for a larger number of 
antennas it is expected that more weight has to be put on spatial multiplexing and less on space-time coding. Interestingly, having a larger number of antennas does not need to result in a larger number of radio frequency $(\mathrm{RF})$ chains. By using antenna selection techniques (see, e.g., [104]-[106]) it is possible to retain the benefits of a large MIMO array with just a subset of antennas being active at the same time.

\section{F. MIMO Systems With Feedback}

One common aspect amongst the algorithms presented above is that they do not require any a priori channel information at the transmitter to extract either transmit diversity of multiplexing gains. Yet, the information theoretic analysis in Section III suggests that additional performance can be extracted from multiple antennas in the presence of channel state information at the transmitter (CSIT) through, e.g., waterfilling. It should be noted that although waterfilling may be optimal from an information theoretic point of view, it is not necessarily the best scheme using CSIT in practice. This is because the performance of real-world MIMO links are sensitive to BER performance rather than mutual information performance. Schemes that exploit CSIT to directly minimize BER-related metrics are therefore of interest, examples of which are found in [107] and [108].

One general drawback of approaches relying on complete and instantaneous CSIT at the transmitter rather than partial or statistical CSIT is feasibility and bandwidth overhead. This makes waterfilling or the equivalent difficult to realize in systems in which the acquisition of CSIT is dependent on a (typically low-rate) feedback channel from RX to TX, such as in frequency-division duplex (FDD) systems. ${ }^{4}$ For a time division duplex (TDD) system feedback is not necessary, but only if the period for switching between a transmitter and a receiver ("ping-pong" time) is shorter than the channel coherence time, which may or may not be realized depending on the mobile's velocity (see Section V). In an effort to bring more performance and robustness to MIMO coding schemes at a reasonable cost of feedback bandwidth, a few promising solutions have been recently proposed to incorporate CSIT in the space-time transmit encoder. Solutions to reduce the feedback cost include using instantaneous yet partial (few bits) CSIT [109] or stastistics of CSIT, such as long term channel correlation information [110], [111], to name a few of the recent papers here.

\section{MiMO ChANNEL Modeling}

Because of the sensitivity of MIMO algorithms with respect to the channel matrix properties, channel modeling is particularly critical to assess the relative performance of the various MIMO architectures shown earlier in various terrains. Key modeling parameters, for which results from measurements of MIMO, as well as SISO can be exploited include path loss, shadowing, Doppler spread and delay spread profiles, and the Ricean $K$ factor distribution. Much more specific to MIMO and, hence, of interest here, are

- the joint antenna correlations at transmit and receive ends;

- the channel matrix singular value distribution. 2000)
In practice, the latter is more accurately represented by the distribution of eigenvalues of $\mathbf{H H}^{*}$, denoted $\left\{\lambda_{1}, \lambda_{2}, \ldots\right\}$. In what follows, we describe the impact of environmental parameters (LOS component, density of scattering) and antenna parameters (spacing, polarization) on the correlation/eigenvalue distribution.

\section{A. Pseudostatic Narrowband MIMO Channel}

1) LOS Component Model: It is common to model a wireless channel as a sum of two components, a LOS component and a NLOS component. That is, $\mathbf{H}=\mathbf{H}_{\mathrm{LOS}}+\mathbf{H}_{\mathrm{NLOS}}$. The Ricean $K$ factor is the ratio between the power of the LOS component and the mean power of the NLOS component.

In conventional SISO wireless deployments, it is desirable that antennas be located where the channel between the transmitter and the receiver has as high a Ricean $K$ factor as possible. The higher the $K$ factor, the smaller the fade margin that needs to be allocated. For example, to guarantee service at $99 \%$ reliability, the fade margin for $K=10$ is more than 10-dB lower than that for $K=0$ (pure Rayleigh fading). Furthermore, as we mentioned earlier, certain beamforming techniques, especially those relying on angle-of-arrival (AOA) estimation are effective only if the LOS component dominates.

For MIMO systems, however, the higher the Ricean $K$ factor, the more dominant $\mathbf{H}_{\mathrm{LOS}}$ becomes. Since $\mathbf{H}_{\mathrm{LOS}}$ is a time-invariant, often low rank matrix [112], its effect is to drive up antenna correlation and drive the overall effective rank down (more precisely the singular value spread is up). High- $K$ channels show low useable spatial degrees of freedom and, hence, a lower MIMO capacity for the same SNR. For example, at $\rho=6 \mathrm{~dB}$, the channel capacity for a $(4,4)$ MIMO channel with $K=0$ is almost always higher than that with $K=10$. Note, however, that this does not mean that one would intentionally place the antennas such that the LOS component diminishes. Near-LOS links typically enjoy both a more favorable path loss and less fading. In such cases, the resulting improvement in link budget may more than compensate the loss of MIMO capacity.

Recently, experimental measurements have been carried out to try to characterize the distribution of the $K$ factor in a coverage area [113]-[115]. In [113], an empirical model was derived for typical macrocell fixed-wireless deployment. The $K$ factor distribution was modeled as lognormal, with the median as a function of season, antenna heights, antenna beamwidth,

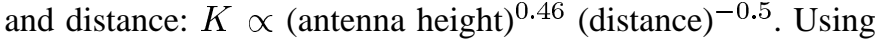
this model, one can observe that the $K$ factor decreases as the distance increases. The implication, from a network deployment perspective, is that even though the use of MIMO does not materially improve the link throughput near the base station, where the signal strength is usually high enough to support the desired applications, it does substantially improve the quality of service in areas that are far away from the base station, or are physically limited to using low antennas.

In metropolitan areas, microcell deployment is popular. In a microcell, the base station antenna is typically at about the same height as street lamp posts, and the coverage radius is no more than a few hundred meters. Microcell channels frequently involve the presence of a LOS component and, thus, may be expected to be Ricean [116]. Similar to macrocells, in a microcell 


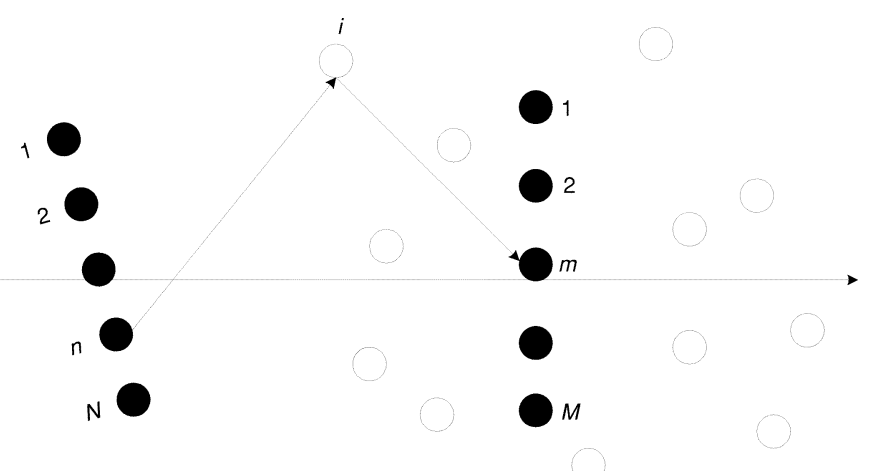

Fig. 10. Diagram to derive antenna correlation $\Psi$. The $i$ th $\left(1 \leq i \leq n_{s}\right)$ path from TX antenna $n$ to RX antenna $m$ goes through the $i$ th single-bounce scatterer.

the $K$ factor declines when distance increases. Overall the $K$ factor observed in a microcell tends to be smaller than that in a macrocell.

In an indoor environment, many simulations [42] and measurements [117] have shown that typically the multipath scattering is rich enough that the LOS component rarely dominates. This plays in favor of in-building MIMO deployments (e.g., WLAN).

2) Correlation Model for NLOS Component: In the absence of a LOS component, the channel matrix reduces to $\mathbf{H}_{\mathrm{NLOS}}$ and is usually modeled with circularly-symmetric complex Gaussian random variables (i.e., Rayleigh fading). The elements of $\mathbf{H}_{\mathrm{NLOS}}$ can be correlated though, often due to insufficient antenna spacing, existence of few dominant scatterers and small AOA spreading. Antenna correlation is considered the leading cause of rank deficiency in the channel matrix, although as we see later, it may not always be so.

Modeling of Correlation: A full characterization of the second-order statistics of $\mathbf{H}_{\mathrm{NLOS}}$ is $\operatorname{cov}\left(\operatorname{vec}\left(\mathbf{H}_{\mathrm{NLOS}}\right)\right) \equiv \Psi$, where cov and vec are the covariance and matrix vectorization operator (stacking the columns on top of each other), respectivelly. In the following, we will introduce commonly accepted models for $\operatorname{cov}(\operatorname{vec}(\mathbf{H}))$. Before that, let us first review a simple model shown in Fig. 10.

Consider a transmitter TX with $N$ antennas and a receiver $\mathrm{RX}$ with $M$ antennas. For simplicity, the antenna pattern is assumed to be omni-directional. Ignoring the rays that involve more than one scatterer, the channel gain between antenna $T_{n}$ and antenna $R_{m}$ is the summation of the contributions from each of the scatterers

$$
h\left(R_{m}, T_{n}\right)=\sum_{i=1}^{n_{s}} r_{i}\left(R_{m}, T_{n}\right)
$$

where $n_{s}$ is the number of scatterers and $r_{i}\left(R_{m}, T_{n}\right)$ is the complex amplitude associated with a ray emanated from antenna $T_{n}$, reflected by scatterer $i$, and then received at antenna $R_{m}$. The correlation between $h_{R_{m}}, T_{n}$ and $h_{R_{m^{\prime}}}, T_{n^{\prime}}$ can then be given by

$$
\begin{aligned}
\Psi\left(R_{m} T_{n}, R_{m^{\prime}}\right. & \left.T_{n^{\prime}}\right) \\
= & \frac{\mathrm{E}\left[\sum_{i=1}^{n_{s}} r_{i}\left(R_{m}, T_{n}\right) \sum_{i=1}^{n_{s}} r_{i}\left(R_{m^{\prime}}, T_{n^{\prime}}\right)^{*}\right]}{\sqrt{\mathrm{E}\left(\left|h\left(R_{m}, T_{n}\right)\right|^{2}\right) \mathrm{E}\left(\left|h\left(R_{m^{\prime}}, T_{n^{\prime}}\right)\right|^{2}\right)}} .
\end{aligned}
$$

An appropriate model for a macrocell deployment in a suburban environment is as follows [118]. The base station TX is elevated above urban clutter and far away from the scatterers, while on the other hand, the mobile terminal RX is surrounded by scatterers. Consider that infinitely many scatterers exist uniformly in azimuth angle around the mobile. Furthermore, consider that the amplitudes of the scattered rays are identical, whereas the phases of them are completely independent. Under these assumptions, one can easily show that $\Psi\left(R_{m} T_{n}, R_{m^{\prime}} T_{n}\right)=J_{0}\left((2 \pi / \lambda) D\left(R_{m}, R_{m^{\prime}}\right)\right)$, where $D\left(R_{m}, R_{m^{\prime}}\right)$ is the distance between antennas $R_{m}$ and $R_{m^{\prime}}$. Hence, the decorrelation distance can be as low as half a wavelength.

It can be more involved to compute the correlation due to antenna separation at the base station, $\Psi\left(R_{m} T_{n}, R_{m} T_{n^{\prime}}\right)$. If the base station is higher than its surroundings, it is often the case that only waves transmitted within azimuth angle $\theta \in[\Theta-$ $\Delta, \Theta+\Delta]$ can reach the mobile. Here, $\Theta$ and $\Delta$ correspond to the AOA and angle spread, respectively. Let us denote the distribution of scatterers in azimuth angle, as seen by the base station, by $p(\theta)$. This function $p(\theta)$ is referred to as a power azimuth distribution (PAD). Given $p(\theta)$, the spatial correlation function can be given by

$$
\begin{aligned}
& \Psi\left(R_{m} T_{n}, R_{m} T_{n^{\prime}}\right) \\
& \quad=\int_{\Theta-\Delta}^{\Theta+\Delta} p(\theta) \exp \left(j \frac{2 \pi \sin (\theta)}{\lambda} D\left(T_{n}, T_{n^{\prime}}\right)\right) d \theta
\end{aligned}
$$

where $D\left(T_{n}, T_{n^{\prime}}\right)$ is the distance between base station antennas $T_{n}$ and $T_{n^{\prime}}$

Let us consider a particular choice of $p(\theta)$ which corresponds to the case where scatterers are uniformly distributed on a circle. The mobile is at the center of the circle. If the mobile is right at the broadside direction, i.e., $\Theta=0$, then $\Psi\left(R_{m} T_{n}, R_{m} T_{n^{\prime}}\right) \approx$ $J_{0}\left((2 \pi \Delta / \lambda) D\left(T_{n}, T_{n^{\prime}}\right)\right)$. On the other hand, if the mobile is at the inline direction, i.e., $\Theta=\pi / 2$, then $\left|\Psi\left(R_{m} T_{n}, R_{m} T_{n^{\prime}}\right)\right| \approx$ $J_{0}\left((2 \pi / \lambda) \Delta^{2} D\left(T_{n}, T_{n^{\prime}}\right)\right)$ [45]. It is apparent that at deployment, to obtain the highest diversity, one must ensure that the orientation of the base station antenna array is such that the mobiles are mostly distributed in the broadside direction. This is already common practice whenever possible. Note that in order for the antenna correlation to be low, one desires a large antenna spacing at the base station; on the other hand, phase-array beamforming will only perform well if the antennas are closely spaced in order to prevent spatial aliasing. Thus, at deployment one must make a choice between optimizing for beamforming or MIMO.

In addition to the PAD chosen above, there are a few other plausible PADs studied in the literature, e.g., uniform, truncated normal and Laplacian [119]. Different PADs naturally leads to different relations between antenna correlation and AOA or angle spread. Nevertheless, all point to the general trend that in order to reduce antenna correlation, one must increase the antenna separation, and ensure that $\Theta$ is as close to zero as possible.

Compared to macrocells, for microcell deployment, the uplink waves arriving at the base station may come predominantly from a few directions. In other words, $p(\theta)$ is nonzero in $\left[\Theta_{0}-\right.$ $\left.\Delta_{0}, \Theta_{0}+\Delta_{0}\right] \cup\left[\Theta_{1}-\Delta_{1}, \Theta_{1}+\Delta_{1}\right] \cup \ldots$ Interestingly, as 


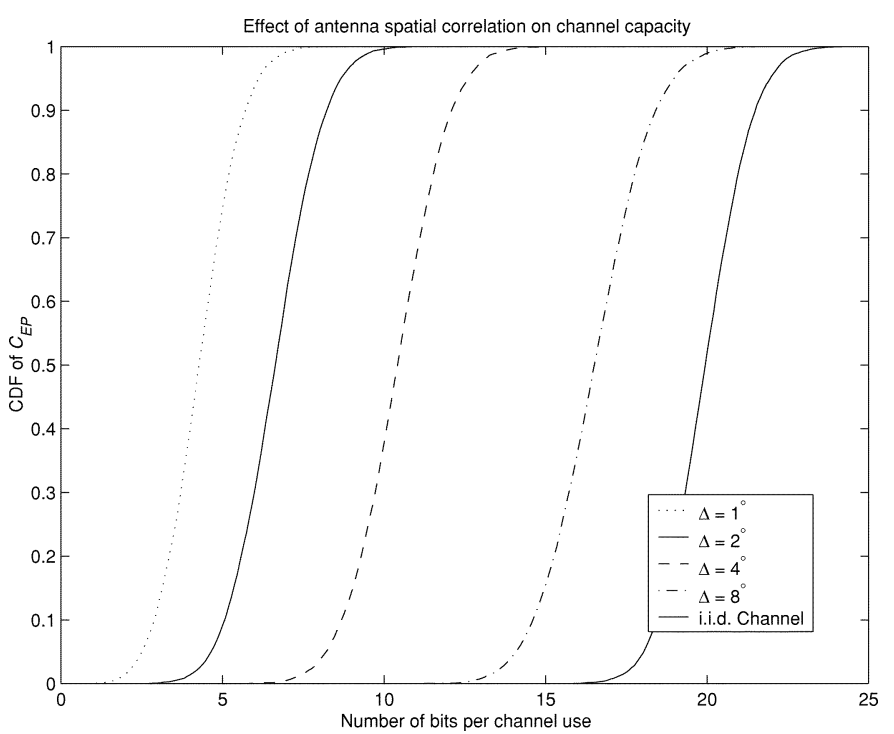

Fig. 11. Distribution of capacity as a function of angle spread for an $(8,8)$ system with $\rho=8$. In producing this figure, transmit power is evenly divided, $\Theta=0^{\circ}, D\left(T_{n}, T_{n^{\prime}}\right)=3 \lambda\left|n-n^{\prime}\right|$, and $D\left(R_{m}, R_{m^{\prime}}\right)=\lambda\left|m-m^{\prime}\right|$.

long as the distribution of $\Theta_{i}$ is diverse enough, the antennas will become fairly uncorrelated, even with angle spreads $\Delta_{i}$ approaching zero [120].

\section{B. Impact of Spatial Correlation}

The statistics of $\operatorname{vec}\left(\mathbf{H}_{\mathrm{NLOS}}\right)$ given $\Psi$ is equal to that of $\Psi^{1 / 2} \operatorname{vec}\left(\mathbf{H}_{w}\right)$, where $\mathbf{H}_{w}$ is an $N$-by- $M$ matrix with i.i.d. circularly symmetric complex Gaussian entries. For convenience, it is common to approximate the correlation matrix $\Psi$ to be a Kronecker product of the two local correlation matrices. That is, let $\Psi^{R}$ and $\Psi^{T}$ denote the antenna correlation matrices at RX (mobile) and TX (base station), respectively; the approximation is $\operatorname{cov}\left(\operatorname{vec}\left(\mathbf{H}_{\mathrm{NLOS}}\right)\right) \equiv \Psi \approx \Psi^{R} \otimes \Psi^{T}$. Under the assumption that the components of $\mathbf{H}_{\mathrm{NLOS}}$ are jointly Gaussian, the statistics of $\mathbf{H}_{\mathrm{NLOS}}$ is identical to those of $\left(\Psi^{R}\right)^{1 / 2} \mathbf{H}_{w}\left(\Psi^{T}\right)^{1 / 2}$. This is a useful form for mathematical manipulation. Fig. 11 shows the distribution of channel capacity of an $(8,8)$ system as a function of angle spread, assuming that the channel statistics can indeed be described by $\left(\Psi^{R}\right)^{1 / 2} \mathbf{H}_{w}\left(\Psi^{T}\right)^{1 / 2}$. In general, as the angle spread becomes narrower, the spatial correlation increases. As a result, the channel capacity decreases.

If the channel $\mathbf{H}$ can be described by $\left(\Psi^{R}\right)^{1 / 2} \mathbf{H}_{w}\left(\Psi^{T}\right)^{1 / 2}$, then an upper bound of channel capacity can be derived. The channel capacity given $\mathbf{H}_{w}$ and an SNR budget $\rho$ can be upper bounded by

$$
C\left(\rho, \mathbf{H}_{w}\right) \leq \max _{\rho_{k}} \sum_{k=1}^{\operatorname{rank}\left(\mathbf{H}_{w}\right)} \log _{2}\left(1+\rho_{k} v_{k}^{R} v_{k}^{T} \lambda_{k}\right)
$$

where $v_{k}^{R}, v_{k}^{T}$ and $\lambda_{k}$ are the $k$ th largest eigenvalues for $\Psi^{R}$, $\Psi^{T}$ and $\mathbf{H}_{w} \mathbf{H}_{w}^{*}$, respectively, and $\sum \rho_{k}=\rho$ [45].

Even though (29) is not a very tight bound, it does offer useful insights into the impact of spatial correlation on channel capacity. The higher the channel correlation, the more rapidly the sequence $v_{k}^{R} v_{k}^{T}$ diminishes toward zero. One can easily obtain an upper bound on the effective channel rank from the products of $v_{k}^{R} v_{k}^{T}$.
1) Decoupling Between Rank and Correlation: Though convenient, one must be careful in using the $\Psi \approx \Psi^{R} \otimes \Psi^{T}$ approximation. For instance a situation can arise where there is significant local scattering around both the BTS and the subscriber unit, causing uncorrelated fading at each end of the MIMO link and yet only a low rank is realized by the channel matrix. That may happen because the energy travels through a narrow "pipe." Mathematically, this is the case if the product of the scattering radius around the transmitter and that around the receiver divided by the TX-RX distance is small compared with the wavelength, as was modeled in [112]. Such a scenario is depicted in Fig. 12. Channels exhibiting at the same time antenna decorrelation (at both ends) and a low matrix rank are referred to as pinhole or keyhole channels in the literature [112], [121]. Pinhole channels can also result from certain rooftop diffraction effects [121]. However, most MIMO measurements carried out so far suggest that rank loss due to the pinhole effect is not common. In fact the results reported largely confirm the high level of dormant capacity of MIMO arrays, at least in urban or suburban environments. Indoor scenarios lead to even better results. Samples of analysis for UMTS type scenarios can be found in [122]-[126]. Measurements conducted at $2.5 \mathrm{GHz}$ for broadband wireless access applications can be found in [115].

2) Correlation Model Between Two Polarized Components: Both reflection and diffraction processes are polarization sensitive, and can produce a rotation of the polarization of the scattered wave compared to the incident wave. This leads to the possibility of constructing a MIMO system using a pair of polarized antennas at both ends, with the two antennas potentially colocated and avoiding some of the issues above related to lack of richness in multipath.

Consider a MIMO channel using a pair of vertical and horizontal polarized antennas at both ends. A $2 \times 2$ matrix with equal-variance complex Gaussian entries clearly is not an appropriate narrowband channel model. First, the propagation environment may dictate that the pass losses for the two polarizations are different. Secondly, the cross-polar component is typically considerably weaker than the co-polar component. In general, the more sparse the scatterers, the lower the effect of cross polarization. Also, as distance between the two terminals increases, the cross polarization decreases. The cross-polarization ratio was found to be around $7.4 \mathrm{~dB}$ in macrocells in the $900 \mathrm{MHz}$ band [127].

In typical outdoor environments with reasonable scattering, it has been found experimentally [127]-[129] that the co-polar and the cross-polar received components are almost uncorrelated. The mean correlation coefficients are around 0.1 or below, and were found to increase somewhat with range in microcells. Nevertheless, as the range increases, the power difference between the co-polar and the cross-polarization components increases. If the difference is high, regardless of the correlation between the co-polar and the cross-polar components, the effective rank of the $2 \times 2$ matrix will always be two.

Overall, the use of multipolarized antenna setups for MIMO opens the door to fairly compact MIMO designs while achieving enhanced robustness with respect to the multipath characteristics [130]. 


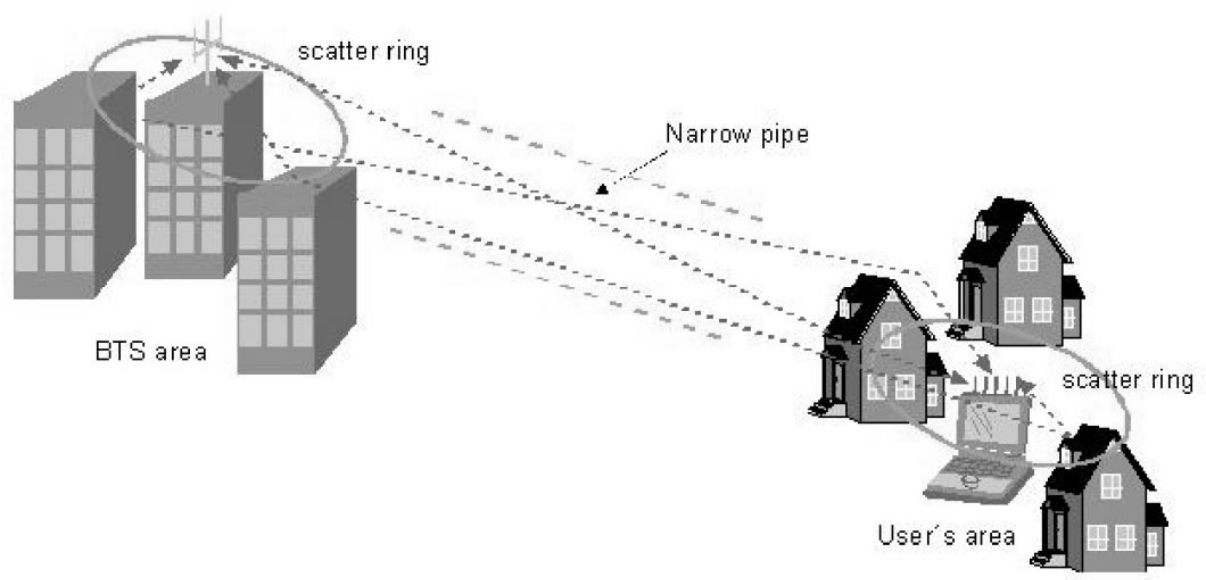

Fig. 12. An example of pin-hole realization. Reflections around the BTS and subscribers cause locally uncorrelated fading. However, because the scatter rings are too small compared to the separation between the two rings, the channel rank is low.

3) Toward Using Orthogonal Antenna Patterns: Antenna pattern diversity at either end of the MIMO link is particularly useful at a site where the waves are coming from diverse angles. Like polarization diversity, it allows for the collocation of antennas. Unlike polarization, where only two orthogonal modes are available, it is theoretically possible to utilize antennas with sharp patterns to obtain many more orthogonal modes. If the incoming waves do indeed distribute uniformly in AOA, a multimode antenna is expected to provide a large number of diversity branches in a very small physical footprint [131], although limited by the number of independent paths.

Since each antenna receives waves coming from different angles, in general one expects the average power, Doppler spectrum, and delay spread profile for each antenna pattern to be different. Thus, to model a MIMO system using antenna pattern diversity correctly, one must be careful in specifying the correlation matrix for $\operatorname{vec}(\mathbf{H})$; a matrix of correlated, equal-variance complex Gaussian entries may not be an appropriate model for such a MIMO channel.

4) Effective Degrees of Freedom: In Section III, we have shown that an $(M, N)$ channel can be decomposed into an equivalent system consisting of $\min (M, N)$ parallel SISO subchannels whose channel power gains are the eigenvalues $\lambda_{k}$ of W. With an SNR so high that $\rho_{k} \lambda_{k}>1 \forall k$, every additional $3 \mathrm{~dB}$ increase in signal power leads to an increase of $\min (M, N) \mathrm{b} / \mathrm{s} / \mathrm{Hz}$ in channel capacity. However, the higher the correlation among the components of $\mathbf{H}$, in general the more widely spaced the primary support regions for the distributions of these eigenvalues. Effective degrees of freedom (EDOF) is a quantity defined to empirically observe the number of these SISO subchannels that effectively contribute to the channel capacity

$$
\left.\mathrm{EDOF} \equiv \frac{d}{d \delta} C_{\mathrm{EP}}\left(2^{\delta} \rho\right)\right|_{\delta=0} .
$$

Although the channel matrix $\mathbf{H}$ has rank $\min (M, N)$ with probability one in general, only the power allocated to EDOF out of these dimensions contributes to channel capacity. EDOF is considered a slowly time-varying property of the channel.

\section{Time-Varying Wideband MIMO Channel}

Similar to the extension of a narrowband SISO channel model to a wideband SISO model, it is generally accepted that one can model a time-varying wideband MIMO channel as a sum of a LOS component and several delayed random fading components

$$
\mathbf{H}(\tau)=\sum_{i=1}^{L} \mathbf{H}_{i} \delta\left(\tau-\tau_{i}\right)
$$

where only $\mathbf{H}_{1}=\mathbf{H}_{\mathrm{LOS}}+\mathbf{H}_{\text {random }}$ contains a LOS component and a random fading component. Note that $\mathbf{H}(\tau)$ is a complex $M \times N$ matrix and $\mathbf{H}_{i}$ describes the linear transformation between the two antenna arrays at delay $\tau_{i}$, possibly using one of the previously mentioned flat fading models. This is simply a tapped delay line model where the channel coefficients at the $L$ delays are represented by matrices. Because the dimension of the antenna array is in general much smaller than the distance light travels between the taps, the short term statistics of these different taps are considered uncorrelated.

As mentioned before, the performance of MIMO techniques depends heavily on the spatial correlation of the antenna elements. For a terminal with limited space resources, MIMO works best when such a terminal is in a location where the decorrelation distance is short. Unfortunately, in such a low decorrelation distance environment, even if the terminal is moving at a reasonable speed, the channel matrix $\mathbf{H}$ can evolve at a very fast rate. This rate is also called Doppler spread and varies from a few Hertz in stationary applications to $200 \mathrm{~Hz}$ or so in fast mobile scenarios.

Clearly, the value of the Doppler spread multiplied by the number of simultaneous users will determine the traffic overhead incurred by channel feedback for cases where a MIMO or STC scheme is implemented that relies on some instantaneous form of CSIT. The Doppler spread also determines the timing requirement from the moment of channel measurement to the moment the transmitter adapts to the channel feedback. A full feedback of CSIT may quickly become prohibitive in practice and simpler rules for transmit adaptation of the MIMO signaling algorithm may be an attractive solution [132]. 
TABLE I

EXAMPLE OF STANDARDIZED MIMO CHANNELS FOR IEEE BODY 802.16

\begin{tabular}{|c|c|c|c|}
\hline \multicolumn{4}{|c|}{ SUI - 1 Channel } \\
\hline & $\operatorname{tap} 1$ & $\operatorname{tap} 2$ & tap \\
\hline Delay $(\mu \mathrm{s})$ & 0 & 0.4 & 0.8 \\
\hline Power $(\mathrm{dB})$ & 0 & -15 & -20 \\
\hline $\mathrm{K}$ factor & 16 & 0 & 0 \\
\hline \multicolumn{4}{|c|}{ Antenna correlation: $\Psi=0.7$} \\
\hline \multicolumn{4}{|c|}{ SUI - 6 Channel } \\
\hline & $\operatorname{tap} 1$ & $\operatorname{tap} 2$ & $\operatorname{tap} 3$ \\
\hline Delay $(\mu \mathrm{s})$ & 0 & 14 & 20 \\
\hline Power $(\mathrm{dB})$ & 0 & -14 & -20 \\
\hline $\mathrm{K}$ factor & 0 & 0 & 0 \\
\hline \multicolumn{4}{|c|}{ Antenna correlation: $\Psi=0.3$} \\
\hline
\end{tabular}

In a location where a LOS component dominates, even if the terminal is moving at a very high speed, the effective change in channel is actually small. Thus, the rate for full channel information feedback can be reasonable.

\section{Standardized Models}

Recently, MIMO models have been standardized in IEEE 802.16 for fixed broadband wireless access and third-generation partnership project (3GPP) for mobile applications. The MIMO channel model adopted in IEEE 802.16 is described in [133]. In [133], a total of six typical models for $(2,2)$ macrocell fixed-wireless channel are proposed. The assumption made in the model includes vertical polarization only, the correlation matrix being the Kronecker product of the local correlation matrices, and every tap sharing the same antenna correlation. Table I shows two of the channel models proposed in [133]. Note that the SUI-1 channel is the most correlated channel and SUI-6 is the least correlated channel.

The discussions in 3GPP [41] are concerned with standardizing MIMO channel models, with the emphasis on definitions and ranges for the following:

- power azimuth spectrum and AOA for macrocells and microcells at zero mobility, pedestrian, and vehicular mobility;

- power delay profiles for the above cases;

- Ricean $K$-factor values for the above cases.

\section{MIMO APPLICATIONS IN 3G WIRELESS SYSTEMS AND BEYOND}

\section{A. Background}

With MIMO-related research entering a maturing stage and with recent measurement campaign results further demonstrating the benefits of MIMO channels, the standardization of MIMO solutions in third generation wireless systems (and beyond) has recently begun, mainly in fora such as the International Telecommunications Union and the 3GPPs. Several techniques, seen as complementary to MIMO in improving throughput, performance and spectrum efficiency are drawing interest, especially as enhancements to present $3 \mathrm{G}$ mobile systems, e.g., high-speed digital packet access (HSDPA) [134]-[136]. These include adaptive modulation and coding, hybrid ARQ, fast cell selection, transmit diversity.

\section{B. MIMO in $3 G$ Wireless Systems And Beyond}

There is little commercial implementation of MIMO in cellular systems as yet and none is currently being deployed for $3 \mathrm{G}$ outside pure transmit diversity solutions for MISO. Current MIMO examples include the Lucent's BLAST chip and proprietary systems intended for specific markets such as Iospan Wireless' AirBurst system for fixed wireless access [137]. The earliest lab trials of MIMO have been demonstrated by Lucent Technologies several years ago.

In the case of 3GPP, some MIMO results are presented here, based on link level simulations of a combination of V-Blast and spreading code reuse [136]. Table II gives the peak data rates achieved by the down link shared channel using MIMO techniques in the 2-GHz band with a $5-\mathrm{MHz}$ carrier spacing under conditions of flat fading. The gains in throughput that MIMO offer are for ideal conditions and are known to be sensitive to channel conditions. In particular, the conditions in urban channels that give rise to uncorrelated fading amongst antenna elements are known to be suitable for MIMO. The gains of MIMO come at the expense of increased receiver complexity both in the base station and in the handsets. Also various factors such as incorrect channel estimation, presence of correlation amongst antenna elements, higher Doppler frequencies, etc., will tend to degrade the ideal system performance. A brief discussion on some of the open issues and remaining hurdles on the way to a full scale commercialization of MIMO systems is contained below.

1) Antenna Issues: Antenna element numbers and interelement spacing are key parameters, especially the latter if the high spectral efficiencies of MIMO are to be realized. Base stations with large numbers of antennas pose environmental concerns. Hence, the antenna element numbers are limited to a modest number, say four, with an interelement spacing of around $10 \lambda$. The large spacing is because base stations are usually mounted on elevated positions where the presence of local scatterers to decorrelate the fading cannot be always guaranteed. Using dual polarized antennas, four antennas can fit into a linear space of $1.5 \mathrm{~m}$ at $10 \lambda$ spacing at $2 \mathrm{GHz}$. For the terminal, $1 / 2 \lambda$ spacing is sufficient to ensure a fair amount of uncorrelated fading because the terminal is present amongst local scatterers and quite often there is no direct path. The maximum number of antennas on the terminal is envisaged to be four, though a lower number, say two, is an implementation option. Four dual polarized patch antennas can fit in a linear space of $7.5 \mathrm{~cm}$. These antennas can easily be embedded in casings of lap tops. However, for handsets, even the fitting of two elements may be problematic. This is because, the present trend in handset design is to imbed the antennas inside the case to improve look and appeal. This makes spacing requirements even more critical.

2) Receiver Complexity: MIMO channel estimation results in increased complexity because a full matrix needs to be tracked per path delay (or per tone in OFDM) instead of a single coefficient. Since practical systems typically limit the number of antenna elements to a few, this added complexity is 
TABLE II

PeAK Data Rates of Various MiMO ARChitectures

\begin{tabular}{l|l|l|l|l|l|l}
\hline$(\mathrm{M}, \mathrm{N})$ & Tx technique & Code rate & Modulation & Rate/sub-stream & \# sub- streams & Data rate \\
\hline$(1,1)$ & Conven-tional & $3 / 4$ & $64 \mathrm{QAM}$ & $540 \mathrm{kbps}$ & 20 & $10.8 \mathrm{Mbs}$ \\
\hline$(2,2)$ & MIMO & $3 / 4$ & $16 \mathrm{QAM}$ & $360 \mathrm{kbps}$ & 40 & $14.4 \mathrm{Mbs}$ \\
\hline$(2,2)$ & MIMO & $3 / 4$ & QPSK & $180 \mathrm{kbps}$ & 80 & $14.4 \mathrm{Mbs}$ \\
\hline$(4,4)$ & MIMO & $1 / 2$ & $8 \mathrm{PSK}$ & $540 \mathrm{kbps}$ & 80 & $21.6 \mathrm{Mbs}$ \\
\hline
\end{tabular}

not seen as a bottle neck. Extra complexity comes from extra $\mathrm{RF}$, hardware, and sophisticated receiver separation algorithms. A MIMO receiver should be dual mode to support non-MIMO mode. In the MIMO mode, it will have multiple RF chains (equal to the number of RX antennas), and additional baseband operations i.e., the space-time combiners and detector to eliminate spatial interference. The additional requirements increase the complexity of a $(4,4)$ MIMO system to about twice that of a single antenna receiver [136], [138], [139]. There may also be additional processing (equalization or interference cancellation) needed due to dispersive channel conditions resulting from delay spread of the environment surrounding the MIMO receiver. The complexity impact of these is not yet fully accounted for.

Homodyne detection may provide direct conversion to baseband and, thus, avoid the need for SAW filters in the IF circuitry. This could reduce the RF complexity aspects of MIMO. Whilst the overall cost impact of MIMO complexity is not clear, one thing is clear: MIMO receivers are likely to cost more than conventional receivers and in the terminal the battery life may also be an issue.

3) System Integration and Signaling: The MIMO system needs to be integrated and be backward compatible with an existing non MIMO network. MIMO signaling imposes the support of special radio resource control (RRC) messages. The terminals need to know via broadcast down link signaling if a base station is MIMO capable. The base station also needs to know the mobile's capability, i.e., MIMO or non-MIMO. This capability could be declared during call set up. Handsets are also required to provide feedback to the base station on the channel quality so that MIMO transmission can be scheduled if the channel conditions are favorable. These downlink and uplink RRC messages are then mapped on to the layer 2 signaling messages [139].

4) MIMO Channel Model: The performance of a MIMO system is very much influenced by the underlying channel model especially the degree of correlation amongst the elements of the channel matrix, delay spread issues, etc. While the propagation models for conventional radio systems have been standardized in [140], there is no agreed MIMO channel model by the ITU as yet.

5) CSI at Transmitter: As shown earlier, the channel capacity is a function of the eigenmodes of the channel. The MIMO capacity will benefit from the transmitter having a knowledge of the channel state and may use water filling instead of equal power allocation [21], [39] or some partial form of feedback. Furthermore, knowing the channel correlation matrix, the transmitter could optimize channel coding, bit allocation per substream in addition to amplifier power management [141]. Various power allocation algorithms are discussed in [36] which are optimum during different channel conditions. The feedback of accurate and timely CSI to the transmitter is another open issue.

\section{CONCLUSIONS AND Future TRENDS}

This paper reviews the major features of MIMO links for use in future wireless networks. Information theory reveals the great capacity gains which can be realized from MIMO. Whether we achieve this fully or at least partially in practice depends on a sensible design of transmit and receive signal processing algorithms. It is clear that the success of MIMO algorithm integration into commercial standards such as 3G, WLAN, and beyond will rely on a fine compromise between rate maximization (BLAST type) and diversity (space-time coding) solutions, also including the ability to adapt to the time changing nature of the wireless channel using some form of (at least partial) feedback. To this end more progress in modeling, not only the MIMO channel but its specific dynamics, will be required. As new and more specific channel models are being proposed it will useful to see how those can affect the performance tradeoffs between existing transmission algorithms and whether new algorithms, tailored to specific models, can be developed. Finally, upcoming trials and performance measurements in specific deployment conditions will be key to evaluate precisely the overall benefits of MIMO systems in real-world wireless scenarios such as UMTS.

\section{ACKNOWLEDGMENT}

The authors would like to thank J. Akhtar for discussions and his help in the simulations. They also would like to thank Prof. D. Falconer, Prof. H. Bolcskei, Prof. A. Paulraj, and Dr. R. Kalbasi and Dr. D. Gore for reviewing the manuscript and for their constructive remarks.

\section{REFERENCES}

[1] G. J. Foschini and M. J. Gans, "On limits of wireless communications in a fading environment when using multiple antennas," Wireless Pers. Commun., vol. 6, pp. 311-335, Mar. 1998.

[2] G. J. Foschini, "Layered space-time architecture for wireless communication in a fading environment when using multielement antennas," Bell Labs Tech. J., pp. 41-59, Autumn 1996.

[3] E. Telatar, "Capacity of multiantenna Gaussian channels," AT\&T Bell Laboratories, Tech. Memo., June 1995.

[4] G. Raleigh and J. M. Cioffi, "Spatial-temporal coding for wireless communications," IEEE Trans. Commun., vol. 46, pp. 357-366, 1998.

[5] H. Bölcskei, D. Gesbert, and A. J. Paulraj, "On the capacity of OFDMbased spatial multiplexing systems," IEEE Trans. Commun., vol. 50, pp. 225-234, Feb. 2002. 
[6] A. Paulraj and C. B. Papadias, "Space-time processing for wireless communications," IEEE Signal Processing Mag., vol. 14, pp. 49-83, Nov. 1997.

[7] A. J. Paulraj and T. Kailath, "Increasing capacity in wireless broadcast systems using distributed transmission/directional reception," Patent 5345599,1994

[8] J. H. Winters, "On the capacity of radio communication systems with diversity in a Rayleigh fading environment," IEEE J. Select. Areas Commun., vol. SAC-5, pp. 871-878, June 1987.

[9] G. D. Golden, G. J. Foschini, R. A. Valenzuela, and P. W. Wolniansky, "Detection algorithm and initial laboratory results using the V-BLAST space-time communication architecture," Electron. Lett., vol. 35, no. 1, pp. $14-15,1999$.

[10] G. J. Foschini, G. D. Golden, P. W. Wolniansky, and R. A. Valenzuela, "Simplified processing for wireless communication at high spectral efficiency," IEEE J. Select. Areas Commun.-Wireless Commun. Series, vol. 17, pp. 1841-1852, 1999

[11] W. Choi, K. Cheong, and J. Cioffi, "Iterative soft interference cancellation for multiple antenna systems," in Proc. Wireless Communications and Networking Conf., Chicago, IL, 2000, pp. 304-309.

[12] D. So and R. Cheng, "Detection techniques for V-BLAST in frequency selective channels," in Proc. Wireless Communications and Networking Conf., 2002, pp. 487-491.

[13] A. Lozano and C. Papadias, "Layered space time receivers for frequency selective wireless channels," IEEE Trans. Commun., vol. 50, pp. 65-73, Jan. 2002.

[14] G. J. Foschini, "Some basic layered space time architectures and their performance," IEEE J. Select. Areas Commun.-Special Issue on MIMO Systems, to be published.

[15] A. Goldsmith, S. Jafar, N. Jindal, and S. Vishwanath, "Fundamental capacity of MIMO channels," IEEE J. Select. Areas Commun.-Special Issue on MIMO Systems, to be published.

[16] R. Blum, J. Winters, and N. Sollenberger, "On the capacity of cellular systems with MIMO," in Proc. IEEE Vehicular Technology Conf., Atlantic City, NJ, Oct. 2001

[17] R. Blum, "MIMO capacity with interference," IEEE J. Select. Areas Commun.-Special Issue on MIMO Systems, 2003, to be published.

[18] S. Catreux, P. F. Driessen, and L. J. Greenstein, "Attainable throughput of an interference-limited multiple-input multiple-output cellular system," IEEE Trans. Commun., vol. 48, pp. 1307-1311, Aug. 2001.

[19] H. Dai, A. Molisch, and H. V. Poor, "Downlink capacity of interference limited MIMO systems with joint detection," IEEE Trans. Wireless Commun., submitted for publication.

[20] J. G. Proakis, Digital Communications. New York: McGraw-Hill, 1989.

[21] I. E. Telatar, "Capacity of multiantenna Gaussian channels," Eur. Trans. Commun., vol. 10, no. 6, pp. 585-595, 1999

[22] J. W. Silverstein, "Strong convergence of the empirical distribution of eigenvalues of large dimensional random matrices," J. Multivariate Anal., vol. 55, no. 2, pp. 331-339, 1995.

[23] P. B. Rapajic and D. Popescu, "Information capacity of a random signature multiple-input multiple-output channel," IEEE Trans. Commun., vol. 48, pp. 1245-1248, Aug. 2000

[24] A. Lozano, F. R. Farrokhi, and R. A. Valenzuela, "Lifting the limits on high-speed wireless data access using antenna arrays," IEEE Commun. Mag., vol. 39, pp. 156-162, Sept. 2001

[25] P. F. Driessen and G. J. Foschini, "On the capacity formula for multiple-input multiple-output wireless channels: A geometric interpretation," IEEE Trans. Commun., vol. 47, pp. 173-176, Feb. 1999.

[26] D. Shiu, Wireless Communication Using Dual Antenna Arrays, ser International Series in Engineering and Computer Science. Norwell, MA: Kluwer, 1999.

[27] A. M. Sengupta and P. P. Mitra, "Capacity of multivariate channels with multiplicative noise: I. Random matrix techniques and large- $n$ expansions for full transfer matrices," Phy. Arch., no. $0010081,2000$.

[28] N. Chiurtu, B. Rimoldi, and E. Telatar, "Dense multiple antenna systems," in Proc. IEEE Information Theory Workshop, 2001, pp. 108-109.

[29] D. Gesbert, N. Christophersen, and T. Ekman, "Capacity limits of dense palm-sized MIMO arrays," in Proc. Globecom Conf., 2002.

[30] S. Wei, D. Goeckel, and R. Janaswami, "On the capacity of fixed length linear arrays under bandlimited correlated fading," in Proc. CISS, Princeton, NJ, Apr. 2002.

[31] A. Edelman, "Eigenvalues and condition numbers of random matrices," Ph.D. dissertation, Mass. Inst. Technol., Cambridge, MA, 1989.

[32] D. Jonsson, "Some limit theorems for the eigenvalues of a sample covariance matrix," J. Multivariate Analysis, vol. 12, pp. 1-38, 1982.

[33] V. L. Girko, Theory of Random Determinants. Norwell, MA: Kluwer, 1990.
[34] - Theory of Linear Algebraic Equations With Random Coefficients. New York: Allerton, 1996

[35] A. T. James, "Distributions of matrix variates and latent roots derived from normal samples," Ann. Math. Statist., vol. 35, pp. 475-501, 1964.

[36] P. J. Smith and M. Shafi, "Waterfilling methods for MIMO systems," in Proc. 3rd Australian Communication Theory Workshop, Canberra, Australia, 2002, AusCTW 2002.

[37] F. R. Farrokhi, G. J. Foschini, A. Lozano, and R. A. Valenzuela, "Linkoptimal space-time processing with multiple transmit and receive antennas," IEEE Commun. Lett., vol. 5, pp. 85-87, 2001.

[38] P. Viswanath, D. N. C. Tse, and V. Anantharam, "Asymptotically optimal water-filling in vector multiple-access channels," IEEE Trans. Inform. Theory, vol. 47, pp. 241-267, 2001.

[39] C. N. Chuah, D. Tse, J. M. Kahn, and R. Valenzuela, "Capacity scaling in MIMO wireless systems under correlated fading," IEEE Trans. Inform. Theory, vol. 48, pp. 637-650, Mar. 2002.

[40] P. J. Smith and M. Shafi, "On a Gaussian approximation to the capacity of wireless MIMO systems," in Proc. Int. Conf. Communications, ICC 2002, 2002, pp. 406-410

[41] "A standardized set of MIMO radio propagation channels," Lucent, Nokia, Siemens, Ericsson, Jeju, Korea, 3GPP TSG-RAN WG1 23, Nov. $19-23,2001$

[42] C.-N. Chuah, J. M. Kahn, and D. Tse, "Capacity of indoor multiantenna array systems in indoor wireless environment," in Proc. GLOBECOM, 98, Sydney, Australia, 1998, pp. 1894-1899.

[43] O. Oyman, R. Nabar, H. Bolcskei, and A. Paulraj, "Lower bounds on the ergodic capacity of Rayleigh fading MIMO channels," in Proc. IEEE GLOBECOM, Taiwan, 2002.

[44] A. Grant, "Rayleigh fading multiple antenna channels," EURASIP $J$. Appl. Signal Processing, pp. 316-329, Mar. 2002.

[45] D. Shiu, G. J. Foschini, M. J. Gans, and J. M. Kahn, "Fading correlation and its effect on the capacity of multielement antenna systems," IEEE Trans. Commun., vol. 48, pp. 502-513, Mar. 2000.

[46] M. Stoytchev, H. F. Safar, A. L. Moustakas, and S. H. Simon, "Compact antenna arrays for MIMO applications," in Proc. IEEE Int. Symp. Antennas and Propagation, 2001, pp. 708-711.

[47] D. Chizhik, F. Rashid-Farrokhi, F. Ling, and A. Lozano, "Effect of antenna separation on the capacity of BLAST in correlated channels," IEEE Commun. Lett., vol. 4, pp. 337-339, Nov. 2000.

[48] L. Hanlen and M. Fu, "Multiple antenna wireless communication systems: Capacity limits for sparse scattering," in Proc. 3rd Australian Communication Theory Workshop, AusCTW 2002, Canberra, Australia, 2002.

[49] H. Ge, K. D. Wong, and J. C. Liberti, "Characterization of multiple-input multiple-output (MIMO) channel capacity," in Proc. IEEE Wireless Communications and Networking Conf. (WCNC), Orlando, FL, 2002.

[50] J. B. Andersen, "Array gain and capacity for known random channels with multiple element arrays at both ends," IEEE J. Select. Areas Commun., vol. 18, pp. 2172-2178, Nov. 2000.

[51] N. Seshadri and J. H. Winters, "Two schemes for improving the performance of frequency-division duplex (FDD) transmission systems using transmitter antenna diversity," Int. J. Wireless Information Networks, vol. 1, pp. 49-60, Jan. 1994.

[52] A. Wittneben, "A new bandwidth efficient transmit antenna modulation diversity scheme for linear digital modulation," in Proc. IEEE ICC'93, vol. 3, Geneva, Switzerland, 1993, pp. 1630-1634.

[53] V. Tarokh, N. Seshadri, and A. R. Calderbank, "Space-time codes for high data rate wireless communication: Performance criterion and code construction," IEEE Trans. Inform. Theory, vol. 44, pp. 744-765, Mar. 1998.

[54] A. Naguib, N. Seshadri, and R. Calderbank, "Increasing data rate over wireless channels," IEEE Signal Processing Mag., vol. 17, pp. 76-92, May 2000.

[55] M. P. Fitz and J. V. Krogmeier, "Further results on space-time codes for Rayleigh fading," in Proc. Allerton, Sept. 1998, pp. 391-400.

[56] Q. Yan and R. S. Blum, "Optimum space-time convolutional codes for quasi-static slow fading channels," in Proc. Wireless Communications and Networking Conf. (WCNC), Sept. 2000, pp. 1351-1355.

[57] S. Baro, G. Bauch, and A. Hansmann, "Improved codes for space-time trellis-coded modulation," IEEE Commun. Lett., vol. 1, pp. 20-22, Jan. 2000.

[58] A. R. Hammons and H. E. Gamal, "On the theory of space-time codes for psk modulation," IEEE Trans. Inform. Theory, vol. 46, pp. 524-542, Mar. 2000.

[59] Z. Chen, J. Yuan, and B. Vucetic, "Improved space-time trellis coded modulation scheme on slow fading channels," in Proc. ISIT, 2001. 
[60] Y. Liu, M. P. Fitz, and O. Y. Takeshita, "A rank criterion for QAM space-time codes," in Proc. IEEE Int. Symp. Information Theory, Dec. 2000, pp. 3062-3079

[61] S. Alamouti, "Space block coding: A simple transmitter diversity technique for wireless communications," IEEE J. Select. Areas. Commun., vol. 16, pp. 1451-1458, Oct. 1998

[62] V. Tarokh, H. Jafarkhani, and A. R. Calderbank, "Space-time block codes from orthogonal designs," IEEE Trans. Inform. Theory, vol. 45, pp. 1456-1467, July 1999

[63] S. Siwamogsatam and M. Fitz, "Improved high rate space-time TCM via orthogonality and set partitioning," in Proc. Int. Symp. Wireless Personal Multimedia Communications, Alborg, Denmark, Sept. 2001.

[64] S. Siwamogsatam and M. Fitz, "Improved high rate space-time TCM via concatenation of expanded orthogonal block codes and MTCM," in Proc. IEEE Int. Conf. Communication, New York, Apr. 2002.

[65] S. Siwamogsatam and M. Fitz, "Improved high rate space-time TCM from an expanded STB-MTCM construction," IEEE Trans. Information Theory, to be published.

[66] N. Seshadri and H. Jafarkhani, "Super-orthogonal space-time trellis codes," in Proc. IEEE Int. Conf. Communication, New York, Apr. 2002.

[67] V. Tarokh, H. Jafarkhani, and A. R. Calderbank, "Space-time block codes for wireless communications: Performance results," IEEE J. Select. Areas Commun., vol. 17, pp. 451-460, Mar. 1999.

[68] G. Ganesan and P. Stoica, "Space-time diversity using orthogonal and amicable orthogonal designs," Wireless Personal Commun., vol. 18, pp. 165-178, Aug. 2001.

[69] H. Jafarkhani, "A quasi orthogonal space time block code," IEEE Trans. Commun., vol. 49, pp. 1-4, Jan. 2001.

[70] O. Tirkkonen, A. Boariu, and A. Hottinen, "Minimal nonorthogonality rate 1 space-time block code for $3+$ tx antennas," in Proc. IEEE Int. Symp. Spread Spectrum Technology, 2000, pp. 429-432.

[71] A. F. Naguib, V. Tarokh, N. Seshadri, and A. R. Calderbank, "A space-time coding based modem for high data rate wireless communication," IEEE J. Select. Areas. Commun., vol. 16, pp. 1459-1478, Oct. 1998

[72] J. K. Cavers, "An analysis of pilot symbol assisted modulation for Rayleigh faded channels," IEEE Trans. Veh. Technol., vol. 40, pp. 683-693, Nov. 1991

[73] S. Sampei and T. Sunaga, "Rayleigh fading compensation method for 16 QAM in digital land mobile radio channels," in Proc. IEEE VTC'89, vol. I, San Francisco, CA, May 1989, pp. 640-646.

[74] M. L. Moher and J. H. Lodge, "TCMP-A modulation and coding strategy for Ricean fading channels," IEEE J. Select. Areas Commun., vol. 7, pp. 1347-1355, Dec. 1989

[75] R. J. Young, J. H. Lodge, and L. C. Pacola, "An implementation of a reference symbol approach to generic modulation in fading channels," in Proc. Int. Mobile Satellite Conf., Ottawa, ON, Canada, June 1990, pp. 182-187.

[76] J. Yang and K. Feher, "A digital Rayleigh fade compensation technology for coherent OQPSK system," in Proc. IEEE Vehicular Technology Conf., Orlando, FL, May 1990, pp. 732-737.

[77] C. L. Liu and K. Feher, "A new generation of Rayleigh fade compensated $\pi / 4$-QPSK coherent modem," in Proc. IEEE Vehicular Technology Conf., Orlando, FL, May 1990, pp. 482-486.

[78] A. Aghamohammadi, H. Meyr, and G. Asheid, "A new method for phase synchronization and automatic gain control of linearly modulated signals on frequency-flat fading channel," IEEE Trans. Commun., vol. 39, pp. 25-29, Jan. 1991

[79] V. Tarokh, S. M. Alamouti, and P. Poon, "New detection scheme for transmit diversity with no channel estimation," in Proc. Int. Conf. Universal Personal Communications, ICUPC '98, Oct. 1998, pp. 917-920.

[80] V. Tarokh and H. Jafarkhani, "A differential detection scheme for transmit diversity," IEEE J. Select. Areas Commun., vol. 3, pp. 1043-1047, July 2000

[81] B. L. Hughes, "Differential space-time modulation," IEEE Trans. Inform. Theory, vol. 46, pp. 145-149, Nov. 2000.

[82] B. M. Hochwald and T. L. Marzetta, "Unitary space-time modulation for multiple antenna communications in Rayleigh flat fading," IEEE Trans. Inform. Theory, vol. 46, pp. 543-564, Mar. 2000

[83] B. M. Hochwald, T. L. Marzetta, T. J. Richardson, W. Sweldons, and R. Urbanke, "Systematic design of unitary spacetime constellation," IEEE Trans. Inform. Theory, vol. 46, pp. 1962-1973, Sept. 2000

[84] J. Yuan and X. Shao, "New differential space time block coding schemes with two three and four transmit antennas," IEEE J. Select. Areas Commun., submitted for publication.

[85] C. Fragouli, N. Al-Dhahir, and S. Diggavi, "Pre-filtered space-time $m$-bcjr equalizer for frequency selective channels," IEEE Trans. Commun., vol. 50, pp. 742-753, May 2002.
[86] A. Naguib, "Equalization of transmit diversity space-time coded signals," in Proc. IEEE Global Telecommunications Conf., 2000. GLOBECOM 'O0, vol. 2, 2000, pp. 1077-1082.

[87] G. Bauch and A. F. Naguib, "Map equalization of space-time coded signals over frequency selective channels," in Proc. IEEE Wireless Communication and Networking Conf. WCNC'99, vol. 1, New Orleans, LA, Sept. 1999, pp. 261-265.

[88] A. Liu, G. B. Giannakis, A. Scaglione, and S. Barbarossa, "Decoding and equalization of unknown multipath channels based on block precoding and transmit diversity," in Proc. Asilomar Conf. Signals, Systems, and Computers, 1999, pp. 1557-1561.

[89] H. Bolcskei and A. Paulraj, "Space-frequency codes for broadband fading channels," presented at the IEEE Int. Symp. Information Theory, Washington, DC, June 2001.

[90] N. Al-Dhahir, "Single-carrier frequency domain equalization for space-time block coded transmission over frequency selective fading channels,” IEEE Commun. Lett., vol. 5, pp. 304-306, July 2001.

[91] E. Lindskog and A. Paulraj, "A transmit diversity scheme for channels with intersymbol interference," in Proc. IEEE ICC'2000, New Orleans, LA, 2000, pp. 307-311.

[92] M. O. Damen, A. Chkeif, and J. C. Belfiore, "Lattice codes decoder for space-time codes," IEEE Commun. Lett., vol. 4, pp. 161-163, May 2000.

[93] S. Verdu, "Multiuser detection," in Advances in Statistical Signal Processing, V. Poor, Ed. Greenwich, CT: JAI, 1993, pp. 369-409.

[94] P. Comon and P. Chevalier, "Source separation: Models, concepts, algorithms and performance," in Unsupervised Adaptive Filtering. ser. Adaptive and learning systems for communications signal processing and control, S. Haykin, Ed. New York: Wiley, 2000, vol. I, Blind Source Separation, pp. 191-236.

[95] J. Cardoso and A. Souloumiac, "Blind beamforming for non-Gaussian signals," IEEE Proceedings, pt. F, vol. 140, pp. 362-370, 1993.

[96] C. Papadias, "A multiuser Kurtosis algorithm for blind source separation," in Proc. Int. Conf. Acoustics, Speech and Signal Processing (ICASSP), 2000, pp. 3144-3147.

[97] P. Loubaton, E. Moulines, and P. Regalia, "Subspace methods for blind identification and deconvolution," in Signal Processing Advances in Wireless and Mobile Communications, G. Giannakis, J. Hua, P. Stoica, and L. Tong, Eds. Englewood Cliffs, NJ: Prentice-Hall, 2001, ch. 3.

[98] A. J. van der Veen and A. Paulraj, "An analytical constant modulus algorithm," IEEE Trans. Signal Processing, vol. 44, pp. 1136-1155, May 1996.

[99] L. Zheng and D. Tse, "Diversity and multiplexing: A fundamental tradeoff in multiple antenna channels," IEEE Trans. Inform. Theory, submitted for publication.

[100] R. Heath and A. Paulraj, "Diversity versus multiplexing in narrowband MIMO channels: A tradeoff based on Euclidian distance," IEEE Trans. Commun., 2001, submitted for publication.

[101] B. Hassibi and B. Hochwald, "High rates codes that are linear in space and time," IEEE Trans. Inform. Theory, vol. 48, pp. 1804-1824, July 2002.

[102] S. Sandhu and A. Paulraj, "Unified design of linear space-time blockcodes," in IEEE GLOBECOM Conf., vol. 2, 2001, pp. 1073-1077.

[103] M. O. Damen, A. Tewfik, and J. C. Belfiore, "A construction of a space-time code based on number theory," IEEE Trans. Inform. Theory, vol. 48, pp. 753-760, Mar. 2002

[104] A. Molisch, M. Z. W. J. Winters, and A. Paulraj, "Capacity of MIMO systems with antenna selection," in Proc. IEEE Int. Conf. Communications, 2001, pp. 570-574.

[105] D. Gore and A. Paulraj, "MIMO antenna subset selection with space-time coding," IEEE Trans. Signal Processing, vol. 50, pp. 2580-2588, Oct. 2002.

[106] R. W. Heath, S. Sandhu, and A. Paulraj, "Antenna selection for spatial multiplexing systems with linear receivers," IEEE Commun. Lett., vol. 5, pp. 142-143, Apr. 2001.

[107] A. Scaglione, P. Stoica, S. Barbarossa, G. B. Giannakis, and H. Sampath, "Optimal designs for space time linear precoders and decoders," IEEE Trans. Signal Processing, vol. 50, pp. 1051-1064, May 2002.

[108] H. Sampath, P. Stoica, and A. Paulraj, "Generalized linear precoder and decoder design for MIMO channels using the weighted muse criterion," IEEE Trans. Commun., pp. 2198-2206, Dec. 2001.

[109] J. Akhtar and D. Gesbert, "Partial feedback based space-time block coding," IEEE Trans. Commun., submitted for publication.

[110] S. Vishwanath, S. Jafar, and A. Goldsmith, "Channel capacity and beamforming for multiple transmit and receive antennas with covariance feedback," in Proc. ICC, Helsinki, Finland, 2001, pp. 2266-2270.

[111] S. Simon and A. Moustakas, "Optimizing MIMO antenna systems with channel covariance feedback," IEEE J. Select. Areas Commun.-Special Issue on MIMO Systems, to be published. 
[112] D. Gesbert, H. Bolcskei, D. Gore, and A. Paulraj, "Outdoor MIMO wireless channels: Models and performance prediction," IEEE Trans. Commun., Dec. 2002.

[113] L. J. Greenstein, S. Ghassemzadeh, V. Erceg, and D. G. Michelson, "Theory, experiments, and statistical models," in Proc. WPMC 99 Conf. , Amsterdam, Sept. 1999

[114] D. S. Baum, D. A. Gore, R. U. Nabar, S. Panchanathan, K. V. S. Hari, V. Erceg, and A. J. Paulraj, "Measurements and characterization of broadband MIMO fixed wireless channels at $2.5 \mathrm{GHz}$," in Proc. ICPWC 2000 , Hyderabad, Dec. 2000, pp. 203-206.

[115] S. Pitchaiah, V. Erceg, D. Baum, R. Krishnamoorthy, and A. Paulraj, "Modeling of multiple-input multiple-output (MIMO) radio channel based on outdoor measurements conducted at $2.5 \mathrm{GHz}$ for fixed BWA applications," in Proc. Int. Conf. Communications, 2002.

[116] E. Green, "Radio link design for microcellular systems," BT Tech. J., vol. 8, no. 1, pp. 85-96, 1990.

[117] R. Stridh, B. Ottersten, and P. Karlsson, "MIMO channel capacity on a measured indoor radio channel at $5.8 \mathrm{GHz}$," in Proc. Asilomar Conf. Signals, Systems and Computers, Oct. 2000, pp. 733-737.

[118] W. C. Jakes, Microwave Mobile Communications. New York: Wiley, 1974

[119] J. Fuhl, A. F. Molisch, and E. Bonek, "Unified channel model for mobile radio systems with smart antennas," in Proc. Inst. Elect. Eng., Radar Sonar Navigation, vol. 145, 1998, pp. 32-41.

[120] P. Driessen and G. J. Foschini, "On the capacity formula for multiple input-multiple output wireless channels: A geometric interpretation," IEEE Trans. Commun., vol. 47, pp. 173-76, Feb. 1999.

[121] D. Chizhik, G. Foschini, and R. A. Valenzuela, "Capacities of multielement transmit and receive antennas: Correlations and keyholes," Electron. Lett., pp. 1099-1100, 2000.

[122] C. C. Martin, J. Winters, and N. Sollenberger, "Multiple input multiple output (MIMO) radio channel measurements," in Proc. IEEE Vehicula Technology Conf., Boston, MA, 2000, pp. 418-421.

[123] J. Ling, D. Chizhik, P. Wolniansky, R. Valenzuela, N. Costa, and K. Huber, "Multiple transmitter multiple receiver capacity survey in Manhattan," Electron. Lett., vol. 37, pp. 1041-1042, Aug. 2001

[124] R. Buehrer, S. Arunachalam, K. Wu, and A. Tonello, "Spatial channel models and measurements for IMT-2000 systems," in Proc. IEEE Ve hicular Technology Conf., May 2001, pp. 342-346.

[125] J. P. Kermoal, P. E. Mogensen, S. H. Jensen, J. B. Andersen, F Frederiksen, T. Sorensen, and K. Pedersen, "Experimental investigation of multipath richness for multielement transmit and receive antenna arrays," in Proc. IEEE Vehicular Technology Conf.-Spring, 2000, pp. 2004-2008.

[126] J. R.-M. L. Schumacher and L. Berger, "Recent advances in propagation characterization and multiple antenna processing in the 3GPP framework," presented at the URSI Radio Conf., Aug. 2002.

[127] S. Kozono, T. Tsuruhara, and M. Sakamoto, "Base station polarization diversity reception for mobile radio," IEEE Trans. Veh. Technol., vol. VT-33, pp. 301-306, 1984

[128] A. M. D. Turkmani, A. A. Arowojolu, P. A. Jefford, and C. J. Kellett, "An experimental evaluation of the performance of two-branch space and polarization diversity schemes at $1800 \mathrm{MHz}, "$ IEEE Trans. Veh. Technol., vol. VT-44, pp. 318-326, 1995.

[129] P. C. F. Eggers, J. Toftgård, and A. M. Oprea, "Antenna systems for base station diversity in urban small and micro cells," IEEE J. Select. Areas Commun., pp. 1046-1057, 1993.

[130] R. Nabar, H. Bolcskei, V. Erceg, D. Gesbert, and A. Paulraj, "Performance of multiantenna signaling techniques in the presence of polarization diversity," IEEE Trans. Commun., pp. 2553-2562, Oct. 2002.

[131] M. Wennström and T. Svantesson, "An antenna solution for MIMO channels: The switched parasitic antenna," in Proc. IEEE Symp. Personal Indoor and Mobile Radio Communication (PIMRC) 2001, San Diego, CA, 2001, pp. 159-163.

[132] S. Catreux, D. Gesbert, V. Erceg, and R. Heath, "Adaptive modulation and MIMO coding for broadband wireless data networks," IEEE Commun. Mag., vol. 40, pp. 108-115, June 2002.

[133] V. Erceg et al., "Channel models for fixed wireless applications," IEEE, Tech. Rep., IEEE 802.16 Work Group, 2001.

[134] 3GPP, "Physical layer aspects of ultra high speed downlink packet access, release 4,", Tech. Rep. 3G TR25.848 v.4.0, 2001-2003.

[135] 3GPP, "Tx diversity solutions for multiple antennas release 5,", Tech Rep. 3G TR 25.869 v 1.0.0, 2001-2006.

[136] 3GPP, "Multiple-input multiple output antenna processing for HSDPA,", Tech. Rep. 3GPP TR 25.876 v0.0.1, 2001-2011.

[137] D. Gesbert, L. Haumonte, H. Bolcskei, and A. Paulraj, "Technologies and performance for nonline-of-sight broadband wireless access networks," IEEE Commun. Mag., vol. 40, pp. 86-95, Apr. 2002.
[138] 3GPP, "Practical aspects of multiple architectures for HSDPA,", Tech. Rep. TSGR1\#16 (00)1219.

[139] 3GPP, "MIMO system integration and signalling in HSDPA,", Tech. Rep. TSGR1\#19(01)0305, Feb. 27-Mar 2, 2001.

[140] "Guidelines for the evaluation of imt 2000, ITU recommendation," ITU, Tech. Rep. M 1225.

[141] 3GPP, "Alternatives in MIMO link design,", Tech. Rep. TSGR1\#19 (01)0333, Feb. 27-Mar. 2, 2001.

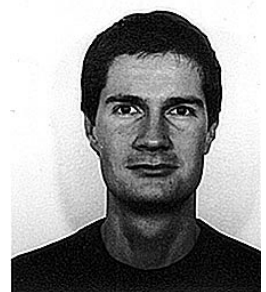

David Gesbert (S'96-M'99) received the Ph.D. degree from Ecole Nationale Superieure des Telecommunications, Paris, France, in 1997.

From 1993 to 1997, he was with France Telecom Research, Paris, where he was involved in the development and study of signal processing algorithms for digital radio communications systems, with emphasis on blind array processing. In 1997 and 1998, he was a Postdoctoral Fellow at the Informations Systems Lab, Stanford University, Stanford, CA. From April 1997 to April 1998, he was the recipient of a French Defense DGA/DRET Postdoctoral Fellowship. In October 1998, he was a cofounder, then a Research Manager at Iospan Wireless, formerly Gigabit Wireless Inc., San Jose, CA, a startup company designing high-speed wireless internet access networks using smart antennas (MIMO), OFDM, and other state-of-the-art applied wireless signal processing research (now Intel). In 2001, he joined the Signal and Image Processing Group, Department of Informatics at the University of Oslo, Oslo, Norway, as an Associate Professor in parallel to his other activities. He has published about 50 conference and journal papers and several patents all in the area of signal processing and communications. His research interests are in the area of signal processing for high-speed digital communications, signal detection, smart antennas and MIMO, multiuser communications, cellular optimization, and resource allocation algorithms.

Dr. Gesbert has served on the Technical Program Committee of various IEEE conferences. He is an Editor for the IEEE TRANSACTIONS ON WIRELESS COMMUNICATIONS.

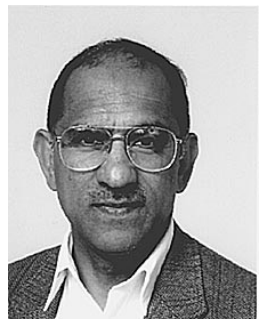

Mansoor Shafi (S'69-A'70-M'82-SM'87-F'93) received the B.Sc. degree in electrical engineering from Engineering University, Lahore, Pakistan, and the Ph.D. degree from the University of Auckland, Auckland, New Zealand, in 1970 and 1979, respectively.

From 1975 to 1979 , he was a Junior Lecturer at the University of Auckland. Since 1979, he has been with Telecom New Zealand, where he holds the position of Principal Advisor Wireless Systems. In 1980, he held a Postdoctoral Fellowship at MacMaster University, Hamilton, ON, Canada. His research interests are in wireless communication systems. He has published widely in many aspects of wireless communications covering radio propagation, signal processing, cellular systems optimization, MIMO systems, etc. He serves as a New Zealand delegate to the meetings of ITU-R that are concerned with the standardization of IMT-2000 systems and beyond.

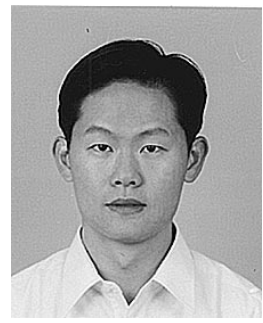

Da-Shan Shiu (S'95-M'99) received the B.S.E.E. degree from National Taiwan University, Taipei, Taiwan, R.O.C., in 1993 and the Ph.D. degree in electrical engineering and computer sciences from the University of California at Berkeley in 1999.

His current research interests include wireless optical communications, space-time signal processing, smart antenna, and spread-spectrum communications. He is currently with Qualcomm Inc., Campbell, CA. His current research interests include wireless optical communications, space-time signal processing, smart antenna, and spread-spectrum communications.

Dr. Shiu is a member of Phi Tau Phi. 


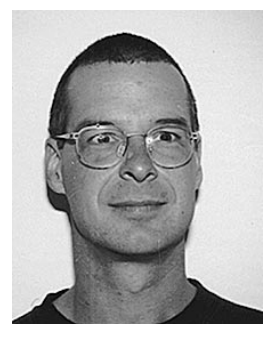

Peter J. Smith (M'93) received the B.Sc. degree in mathematics and the Ph.D. degree in statistics from the University of London, London, U.K., in 1983 and 1988 , respectively.

From 1983 to 1986, he was with the Telecommunications Laboratories, GEC Hirst Research Centre, Wembley, U.K. From 1988 to 2001, he was a Lecturer in statistics and Consulting Statistician at Victoria University of Wellington, Wellington, New Zealand. He is currently a Senior Lecturer in the Department of Electrical and Computer Engineering, University of Canterbury, Christchurch, New Zealand. His research interests include the statistical aspects of design and analysis for communication systems, especially MIMO systems, antenna arrays, and mobile radio.
Ayman Naguib (S'91-M'96-SM'00) received the B.Sc. degree (with honors) and the M.S. degree in electrical engineering from Cairo University, Cairo, Egypt, in 1987 and 1990, respectively, and the M.S. degree in statistics and the $\mathrm{Ph} . \mathrm{D}$. degree in electrical engineering from Stanford University, Stanford, CA, in 1993 and 1996, respectively.

From 1987 to 1989 , he spent his military service at the Signal Processing Laboratory, The Military Technical College, Cairo. From 1989 to 1990, he was a Research and Teaching Assistant in the Communication Theory Group, Cairo University. From 1990 to 1995, he was a Research and Teaching Assistant in the Information Systems Laboratory, Stanford University. In 1996, he joined AT\&T Labs, Florham Park, NJ, as a principal member of technical staff, where he was a Member of the research team in AT\&T Labs that pioneered the field space-time coding. In September 2000, he joined Morphics Technology, Inc., as a Technical Leader and Manager of Core Technology. In October 2002, he joined Qualcomm, Inc., Campbell, CA, where he is a Senior Staff Engineer working future wireless systems. His current research interests include space-time coding, signal processing, and coding for wireless communications. He holds five U.S. patents and seven other pending patent applications in the area of space-time coding and signal processing.

Dr. Naguib is currently serving as an Associate Editor for CDMA and space-time systems, IEEE TRANSACTIONS ON COMMUNICATIONS. 